\title{
A COMPRA DIRECTA DE OBRAS DE ARTISTAS VASCOS CONTEMPORÁNEOS POR LOS MUSEOS PÚBLICOS DEL PAÍS VASCO Y EL IMPACTO SOBRE EL PRECIO DE SUS OBRAS EN LAS SUBASTAS DE ARTE: 1985-2017
}

\author{
XESQUI CASTAÑER LÓPEZ \\ Departament d'Història de l'Art. Universitat de València († 1/10/2018) \\ JOSÉ LUIS HERNÁNDEZ-MARCO
}

Departamento de Historia e Instituciones Económicas. Universidad del País Vasco/EHU (jubilado)

\section{Introducción}

Cuando Sotheby's compró en Octubre de 2016 el Índice de Precios de obras pictóricas de Mei y Moses, publicado originariamente en (2002), denominándolo Sotheby's Mei Moses, si no los inversores y los coleccionistas de arte, sí al menos el gran público ${ }^{1}$ tomó conciencia de la existencia de uno de los asuntos más prolíficos de la Economía de la Cultura: los ejercicios econométricos para analizar el comportamiento histórico de los precios de las obras de arte y sus determinantes, fundamentalmente, mediante el uso de bases de datos procedentes de las casas de subastas.

El primer objetivo de esos ejercicios fue analizar cómo los precios de las subastas pueden ser usados para analizar los movimientos en el mercado del arte y compararlos con otros mercados, habitualmente de acciones y bonos. En la que, probablemente, es la mejor síntesis bibliográfica sobre la materia Orley Ashenfelter y Kathry Graddy (2003), además de construir sus propios índices sobre impresionistas y arte moderno, reseñaron 19 trabajos que, entre 1974 y 2001, habían estimado el rendimiento financiero de las inversiones en Arte mayoritariamente utilizando metodologías hedónicas y la de ventas repetidas. Como indican es- tos autores, dada la idiosincrasia de las obras de arte, la mayoría de los índices construidos se basan en un modelo en donde el precio del objeto $i^{\text {th }}$ vendido en el periodo de tiempo $t$ es

$$
p_{i t}=p_{i}+p_{t}+\varepsilon_{i t}
$$

en donde $p_{i}$ es el componente fijo del precio que refleja el carácter único y fijo (o "calidad") del objeto; $p$ refleja los movimientos de los precios agregados; y $\varepsilon_{i t}$ es el término de error. Igualmente escriben que el punto clave es cómo se trata el componente fijo del precio. Si el método de ventas repetidas lo controla explícitamente, los modelos hedónicos lo hacen mediante un número de características que determinan dicho precio implícito. Como indica la literatura económica sobre los bienes de consumo duraderos (Gordon, 1990, págs. 89-90) la regresión hedónica asume que el precio observado de un producto en un tiempo dado $\left(P_{k t}\right)$ es una función de sus características cualitativas $(x)$, muy a menudo especificadas en forma semilogarítmica:

$$
\ln \left(P_{k t}\right)=\beta_{0}+\sum \beta_{j} x_{j k t}+\varepsilon_{k t}
$$

El problema empírico de la estimación de esas características cualitativas determinantes del precio

1 El British Rail Pension Fund comenzó a invertir en arte ya en 1974 y Philip Hoffman fundó en 2001 el Fine Art Group que lanzó sus Fine Art Fund (I, II y III) en 2004, 2006 y 2010 y en 2013 el Fine Art Guarantee Fund. (Morgan Stanley, n.d.), Por su parte, Deloitte Louxembourg y ArtTactic, publicaron en 2011 su primer "Art \& Finance Report y en el referente a 2016 estiman en US\$ 1.20 billones los "Assets under Management" de los fondos de inversión en arte. (DELOITTE LUXEMBOURG AND ARTTACTIC, 2017). Las referencias periodísticas a la compra del Índice de Mey y Moses son muy numerosas y en la línea de (GALL, 2016) o (POGREIN, 2017). 
de una obra de arte $\left(x_{j k t}\right)$ ha ido derivando hacia incrementar el número de características medibles e, incluso, a trabajos que se centran en ello exclusivamente, es decir, que analizan los determinantes de los precios más que en el rendimiento de la inversión en arte. Una búsqueda en la base de datos Econlit, con los términos "art auction prices" realizada en Noviembre de 2017, contiene más de 90 trabajos académicos nuevos desde la síntesis citada más arriba, ampliando tanto geográfica como cronológicamente las obras de arte, así como las características medibles de las mismas, los periodos de análisis e incluso, las metodologías de cálculo.

Este trabajo continua estas últimas tendencias pues se centra en los determinantes de los precios de las obras de artistas vascos nacidos en los siglos XIX y $X X$, estilísticamente modernos y/o contemporáneos y amplía el número de características medibles de las obras rematadas en subastas entre 1985 y 2017, novedosamente incorporando el papel que las compras directas de los museos -también vascosen los mercados primario y secundario principalmente, pueden tener en los precios que las obras de esos artistas alcanzan en el mercado terciario de las subastas.

Los museos, como "non profit enterprises" (Gassler \& Grace, 1980) o, incluso, como "productive organisations" (Jhonson \& Thomas, 1998) han sido también objeto de estudio para la economía y la administración de empresas. Varios encuentros internacionales de administradores de museos y economistas han tenido lugar a lo largo del tiempo, haciéndose públicos sus resultados: en 1991 en el NBER (Feldstein, 1991), en la Universidad de Durham en 1998 (Volumen 22, Issues 2\&3 del Journal of Cultural Economics, 1998), en Helsinki (Uusitalo, Liisa (ed), 2008) o, más recientemente, las seis Conferencias habidas entre 2005 y 2017 de la AIMAC (Asociación Internacional de la Gestión de las Artes y la Cultura). ${ }^{2}$

Como indica John W. O'Hagan (1998), si bien la funciones de un museo de arte están íntimamente interrelacionadas, de la primera de ellas, la "collection or acquisition" dependen todas las demás pues si no hay colección, no es posible que haya conservación, ni estudio/investigación, ni interpretación/educación, ni exposición/comunicación. Por eso los directivos de los museos consideran que el crecimiento de la colección es el principal de sus objetivos (Cantor, 1991, pág. 18). Sin embargo, lo que constituye la mayor parte de la riqueza de una institución como en el museo de arte, la colección, no aparece en sus cuentas anuales y sus informes públicos no hacen referencia al precio de sus obras. (Frei \& Meier, 2006, págs. 1027-1028). William D. Grampp reflexionó sobre esta ocultación en varios trabajos, interpretando que para los responsables de los museos esta ocultación tiene su origen tanto en la posición antimercantilista de muchos responsables museísticos - "because the inmeasurable cannot be measured" (2007, pág. 249) como en la creencia de que publicitar el valor de su colección puede desanimar donaciones privadas y subvenciones públicas para incrementarla (1991, págs. 172173), aunque los museos guarden una detallada relación de sus colecciones (1991, pág. 170).

Afortunadamente, los cuatro principales museos exclusivamente públicos del País Vasco, ubicados en las tres capitales provinciales y principales ciudades -Bilbao, San Sebastián y Vitoria-Gasteizguardan esa detallada relación de sus colecciones y si bien no publican todos los datos que contienen las mismas, nos permitieron su consulta directa en los años del cambio al actual siglo.

Por ello, nuestro trabajo va a utilizar, como detaIlaremos en la próxima sección, dos bases de datos distintas. Una, de las subastas de obras de artistas vascos y otra, de las obras de esos mismos artistas en los museos vascos y del precio que estos museos pagaron por ellas si las adquirieron en cualquiera de los mercados primario -compra a los propios artistas o sus herederos-, secundario -coleccionistas y galerías- o terciario -subastas-. La utilización conjunta de ambas bases de datos nos permitirá profundizar en la línea en que finalizaba el clásico trabajo de Pommerehne y Field sobre el impacto de las compras de los museos en los precios de las subastas de pinturas:

Once the various influences are disentangled and isolated successfully, other hypotheses that been mentioned superficially, now lend themselves to empirical examination. For instance, (...) it would be of interest to examine the statement about the reversed causality, which has it that the acquisition of an object of a specific painter by a museum will have a significant positive impact on the auction prices to be retrieved thereupon for the other paintings. (La cursiva es nuestra) (Pommerehne \& Feld, 1997, p. 265)

Con el principal objetivo de determinar ese significativo impacto de las adquisiciones de los museos en las subastas de otras obras del mismo artista, en la Sección 2 de este trabajo, describiremos las dos bases de datos empleadas; en la Sección 3, ex-

${ }^{2}$ https://www.gestiondesarts.com/fr/aimac/conferences/\#.WiUwpOribGg 
plicaremos el análisis multinivel utilizado; en la 4 mostraremos los resultados empíricos obtenidos y, finalmente, señalaremos algunas conclusiones.

\section{Descripción de las Bases de Datos}

\section{Los museos vascos y la configuración de sus colecciones}

La creación de los Museos del País Vasco y la conformación de sus colecciones, en el contexto de la de los museos españoles y de las específicas ventajas económico-fiscales del País Vasco (y Navarra) -por el "Concierto Económico y el Cupo"- ha sido tratada en anteriores trabajos (Castañer López \& Hernández Marco, 2015). Baste señalar aquí que el nacimiento de los museos públicos españoles se suele hacer coincidir con la apertura al público en 1819 del Real Museo de Pinturas y Esculturas en el actual edificio del Museo del Prado de Madrid. Fernando VII creó el museo con una colección de algo más de 1.800 obras, de las que se expusieron 310 procedentes de los Reales Sitios. Como efectos colaterales de las desamortizaciones eclesiásticas y las primeras normas sobre protección del patrimonio nacional de 1836/37 se funda, también en Madrid, el llamado Museo de la Trinidad en 1838, formado con 554 pinturas y muchos otros objetos, de conventos suprimidos en las provincias de Madrid, Ávila, Burgos, Segovia y Toledo, más los bienes incautados al infante D. Sebastián Borbón y Braganza. Una cierta azarosa vida de este museo, al vaivén de las coyunturas políticas, lo acabaría suprimiendo e integrando su colección en la del ya Museo Nacional del Prado en 1870 y 1872. Con algunas modificaciones, el proceso inicial de formación de la colección del Museo de la Trinidad, con el principio burgués de considerar nacional el patrimonio eclesiástico desamortizado y el de la propia corona, se procuró extender fuera de Madrid con la R.O. de 13 de junio de 1844, que posibilitaría el nacimiento de muchos Museos Provinciales y/o Municipales, también con las colecciones iniciales originadas en los conventos suprimidos de las respectivas provincias (Antigüedad del Castillo-Olivares, 1998). Este origen desamortizador y burgués es también rastreable en los antecedentes museísticos de los museos vascos.

Pero frente al resto de las administraciones provinciales y locales españolas y sus posibles instituciones museísticas de ellas dependientes, las vascas (y también navarras) tienen la importante particularidad de la foralidad y su corolario del Concierto Económico. Esto es, por una parte, la de contar con unos mayores recursos presupuestarios, al recaudar los impuestos y "descontar" el Cupo a pagar al Estado -tras negociarlo bilateralmente- y, por otra, el poder gestionar el resto en función de sus propias políticas económicas, sociales y culturales (Alonso Olea, 1995 y Vicario y Peña \& Alonso Olea, 1997). Además, las muy diferentes coyunturas políticas españolas durante el siglo XX afectaron desigualmente a las tres provincias, perdiendo esa capacidad foral las "traidoras" Guipúzcoa y Vizcaya durante el franquismo, mientras que la mantendrá durante todo el siglo ininterrumpidamente la "leal" Álava (Badía Lacalle, 1975).

En Bilbao, hay un antecedente en la línea ya comentada anteriormente: la creación de un Museo de Pinturas de Vizcaya en 1842 con el arrendamiento de una casa por la Diputación en 1845 para albergarlo, que llegó a contener treinta pinturas.

Pero, parece existir un consenso en asociar a la promesa del ingeniero de minas D. Laureano de Jado, de donar su colección "al Museo de Bilbao", si se creara, o hacer su donación a otra institución fuera de Vizcaya, como la espoleta que movió realmente a su concreción, eso sí, en una ambiente propicio en la burguesía bilbaína y sus representantes políticos. De este modo, el Museo de Bellas Artes de Bilbao nace oficialmente el 5 de octubre de 1908, cuando la Diputación Foral y el Ayuntamiento bilbaíno crean el Patronato del Museo. Inicialmente se instaló -compartiendo uso-, en el edificio neoclásico de lo que había sido el Hospital Civil de Achuri. Al Museo se le asignó un patio acristalado que permitió configurar tres salas. A ellas se trasladaron para su exposición, obras propiedad de la Diputación; algunas de las de aquel antiguo Museo de Vizcaya; del Ayuntamiento, procedentes del Consulado y Casa de Contratación; de la Escuela de Artes y Oficios; de la Casa de Juntas de Guernica; junto con algunas cedidas por el mencionado Jado, Antonio Plasencia y el primer Director, el pintor Juan Losada. Desde el origen, pues, coleccionismo y gustos artísticos mixtos público-privados. Con esos fondos y ubicación, se inauguró el 8 de febrero de 1914.

El éxito de una iniciativa cultural de la Diputación de Vizcaya, la celebración en 1919 de una "Exposición Internacional de Pintura y Escultura" moderna reforzaría el papel público institucional, representante de la burguesía minera, industrial y comercial vizcaína, en la promoción y difusión también del Arte Moderno. En 1922, y aduciendo igualmente a razones del poco espacio disponible en el de Bellas Artes, la Diputación creó un Museo de Arte Moderno, que abrió sus puertas en 1924, instalado en la segunda planta de un edificio de 
la Diputación, donde se trasladó la obra de los pintores vascos vivos del Museo de Bellas Artes y las obras adquiridas en la Exposición Internacional de 1919. Ambos museos coexistieron pues, hasta la guerra civil española, cuando el almacenaje de la colección del de Bellas Artes y la expatriación de la del de Pintura Moderna, solo permitieron cuando se volvieron a reunir ambas, casi en su totalidad, mantenerlas almacenadas en el Depósito Franco de Uribitarte en el puerto, pues los edificios originales estaban ahora dedicados al esfuerzo bélico. Por ello, el 3 de febrero de 1939 -de nuevo la Diputación y el Ayuntamiento, ambos ya plenamente franquistas- acordaron la financiación para la construcción de un edificio para Museo de Bellas Artes, en un terreno cedido por el Ayuntamiento en el Parque de $D^{a}$ Casilda, entonces denominado de las "Tres Naciones". El Museo ya definitivo, que tuvo sendas importantes ampliaciones en 1963 y 1984/86, se inauguró fusionando los patronatos y colecciones de los dos previos, el 17 de junio de 1945 (Luna, 1989 y Zugaza Miranda, 1999).

Más local que provincial es el caso del donostiarra Museo Municipal de San Telmo. Posiblemente, su titularidad municipal, esté ligada al crecimiento de la propia ciudad, al carácter de la industrialización guipuzcoana y al fuerte incremento demográfico, bastante homogéneo, de otros centros urbano-industriales de la provincia. Pero San Sebastián se configura desde la Restauración como un centro turístico de primer orden y tendrá que dotarse de infraestructuras comerciales y culturales que consoliden esa función (Castells, 1987). Así, a iniciativa de la Sociedad Económica Bascongada de Amigos del País, el Ayuntamiento aprobó la creación del Museo Histórico, Artístico y Arqueológico. Con una pequeña colección, producto fundamentalmente de donaciones, se inauguró el 5 de octubre de 1902 en un edificio municipal, compartiendo la mitad de la planta baja con el Instituto Libre. En 1905, el Ayuntamiento comenzó a construir un edificio, en la calle Urdaneta, para sede compartida de la Escuela de Artes y Oficios, la Biblioteca Municipal y el mismo museo, donde permanecería desde 1911, cuando fue inaugurado, hasta 1932. Ese año se trasladó, junto a la Biblioteca Municipal, con la que convivió hasta 1951, a un antiguo convento de dominicos, restaurado y adquirido por el ayuntamiento en 1928, tomando popularmente el nombre antiguo del convento, San Telmo. En él también tuvo su sede hasta 1994 la promotora inicial del proyecto museístico en 1900, la Sociedad Bascongada de Amigos del País, junto a muchas otras y diversas asociaciones culturales (Nicolau \& Zulaika, 2005).
Como se acaba de ver, el origen y primeros pasos de los museos de Bilbao y San Sebastián se acompasa a las industrializaciones vizcaína y guipuzcoana y el afianzamiento de sus respectivas burguesías industriales y comerciales en las respectivas capitales provinciales, con el añadido, en el caso donostiarra, de su función como centro turístico y de ocio para las élites político-sociales de otras partes de España, especialmente de Madrid.

No es de extrañar, pues, el atraso de la rural Álava y su "levítica" capital Vitoria (Rivera Blanco, 1992), también en estas manifestaciones cultural-museísticas protagonizadas por los representantes políticos de la burguesía industrial y comercial, mucho más potentes en las vecinas provincias. Aunque en Vitoria, como vimos en Bilbao, llegó a formarse a medados del siglo XIX (1844 y 1866) una colección pública y un museo con algunas obras procedentes de la desamortización eclesiástica, pero que solo duró un año o no llegó a nacer, a diferencia de sus capitales hermanas, se retrasaría su continuidad hasta después de la Guerra Civil, cuando comience la acelerada industrialización alavesa, el desarrollismo antes del desarrollismo, como ha sido definido este proceso (García Zúñiga, 2009). Sin embargo, también empiezan a surgir algunas iniciativas que comenzarán a posibilitar la existencia futura, aunque con retraso, de esa infraestructura cultural. En 1827 se reanudan las clases de dibujo en la Academia de Arte que se había creado a finales del siglo XVIII a iniciativa de la Real Sociedad Bascongada de Amigos del País. En 1891, ante la insuficiencia de espacio, se construye un edificio de dos plantas al que se denomina Academia de Bellas Artes. En 1923 se construye el actual edificio para la Escuela de Artes y Oficios. Consecuencia de estos centros de enseñanza artística se realizan algunas exposiciones. Así, en 1916 el Real Ateneo de Vitoria organiza una exposición con la participación de 17 pintores y dibujantes. En 1934, en el Paraninfo de la Escuela de Artes y Oficios se celebra una exposición homenaje al pintor Ignacio Díaz de Olano y sus discípulos. Dos años más tarde en 1936, en el mismo marco, se organiza la Exposición de Pintura de Artistas Alaveses, posiblemente la muestra de pintura alavesa más importante de este siglo, en la que participan 19 pintores con 100 obras. Pero será solo en 1941, cuando la Diputación Foral decide comprar el Palacio de Augusti -hotel particular construido en 1912 por Ricardo Augusti, Conde de Dávila y promotor inmobiliario-, con el objetivo de albergar, en un principio, las colecciones de los Museos de Arte y Arqueología, el Archivo y la Biblioteca Provinciales, así como las colecciones de la Escuela de Artes y Oficios y el Insti- 
tuto de Segunda Enseñanza, hoy sede del Parlamento Vasco. A partir de 1966, se individualizan las diversas colecciones -incluso se instala un Museo de Arte Vasco en la también adquirido palacio de Ajuria-Enea, frente al anterior, hasta su cesión como sede del Gobierno Vasco-y, con la creación del Museo de Arqueología, se gestó la individualización del Museo de Bellas Artes de Álava, de la que posteriormente, con su inauguración en el año 2002, se segregó la parte más contemporánea de su colección para la creación del Artium (Begoña, et al., 1982 y González de Aspuru \& Sancristóval, 2001).

\section{a. La base de datos de las compras museísticas}

Los cuatro museos, así creados y configuradas sus iniciales colecciones, tenían al principio del siglo XXI 4.862 obras (sin contar la obra gráfica y las de arte decorativo). Para tener una mayor base estadística de las obras compradas hemos añadido a las anteriores, 1.127, adquiridas directamente por las Diputaciones Forales de Vizcaya (708) y Guipúzcoa (419) que, en su mayor parte, se exhiben o están depositadas en espacios públicos, especialmente en sus respectivos museos de Bilbao y San Sebastián. Del total de estas 5.989 pinturas y esculturas, $2.733(46 \%)$ ingresaron en las instituciones mediante compra directa entre 1900 y 2002. De estas compras, solamente $55(2 \%)$ se realizaron en el mercado terciario, es decir, en subastas. La inmensa mayoría de las compras se realizaron en el mercado primario -compra directa al artista o a sus familiares-, 696 (25.5\%) y, especialmente, en el secundario -a coleccionistas y galerías- 1.982 (72.5\%).
De estas últimas 2.733 obras en los inventarios consultados -actualizados hasta 2002 para el museo de Bilbao/Diputación de Vizcaya y los dos de Vitoria-Gasteiz y hasta 1999 el de San Sebastián/ Diputación de Guipúzcoa-, consta el precio de costo de 2.330 , de las que 1.128 fueron realizadas por artistas vascos o que realizaron allí buena parte de sus obras, lo que en adelante denominaremos artistas vascos, nacidos en los siglos XIX y XX. Por tanto, nuestra primera base de datos se refiere a 1.128 obras de artistas vascos contemporáneos, de las que conocemos el año de compra por los museos/ Diputaciones -512 de Vizcaya, 460 de Álava y 156 de Guipúzcoa-, su precio de costo y, en la mayoría de los casos, a quién o cómo se adquirió. Las 1.128 obras fueron realizadas por 421 artistas vascos contemporáneos, pero una gran parte de ellos son muy desconocidos fuera del estricto ámbito de alguna de las tres provincias vascas. Por esta razón, hemos seleccionado a los 91 que hemos considerado más conocidos del arte vasco de los siglos XIX y $X X$. Todos ellos tienen al menos tres obras, de las que conocemos el precio, en las adquisiciones de las colecciones públicas de más de una provincia y, además, les ha sido subastada alguna obra entre 1985 y 2017 según nuestra segunda base de datos.

El Apéndice 1 contiene la relación completa de los artistas y sus datos más significativos. En conjunto, estos 91 artistas tienen 1,.262 obras en las colecciones, de las que 773 fueron compradas desde 1912 a 2002 , significando el $68.5 \%$ de todas las obras de artistas vascos adquiridas. Salvo 10 obras que se compraron en el mercado terciario de las subastas, las 763 restantes se compraron en los mercados primario y secundario.

Tabla 1

BASE DE DATOS DE LOS MUSEOS. ESTADÍSTICA DESCRIPTIVA (EN EUROS CONSTANTES DE 2010)

\begin{tabular}{|c|c|c|c|c|c|c|}
\hline & TOTAL & MIN. & MÁX. & MEDIA & MEDIANA & DESV. EST \\
\hline Artistas & 91 & & & & & \\
\hline Artistas "pre-actuales" & 47 & & & & & \\
\hline Año de nacimiento & 91 & 1831 & 1973 & 1908 & 1907 & 35,35 \\
\hline Año de fallecimiento & 64 & 1906 & 2014 & 1964 & 1966 & 30,04 \\
\hline Vivos en 2017 & 27 & & & & & \\
\hline Catalogados on-line & 75 & 1 & 34 & 6,88 & 3 & 7,87 \\
\hline Obras en los museos & 1.262 & 3 & 75 & 13,87 & 11 & 11,02 \\
\hline Obras totales compradas & 773 & 1 & 31 & 8,49 & 7 & 6,21 \\
\hline Compradas en 1901-1936 & 87 & & & 2,42 & & \\
\hline Compradas en 1937-1976 & 160 & & & 4 & & \\
\hline Compradas en 1977-2002 & 526 & & & 21,04 & & \\
\hline $\begin{array}{l}\text { Precio medio de las } \\
\text { Compras por artista }\end{array}$ & & 1.585 & 178.718 & 23.462 & 10.900 & 29.251 \\
\hline Costo total por artista & 25.794 .912 & 3.006 & 5.540 .262 & 283.461 & 68.046 & 671.362 \\
\hline
\end{tabular}


Nacieron en el siglo XIX, 41 de ellos -3 en su $2^{\circ}$ cuarto, 16 en el $3^{\circ}$ y 22 en el $4^{\circ}-$ y en el siglo $X X$ los restantes $50-14$ en el primer cuarto de la centuria, 21 en el $2^{\circ}$ y 15 en el $3^{\circ}$-. En el listado aparecen 27 autores vivos al finalizar 2017 y 64 ya fallecidos -20 en la primera mitad del siglo XX, 21 entre 1950 y 1975, 14 en el último cuarto del siglo XX y los restantes 9 en lo que llevamos de siglo XXI-.

Estilísticamente podemos considerar modernos o pre-actuales a 47 y con influencias de los movimientos artísticos desde la II GM hasta la actualidad, los "contemporáneos" o del Arte Actual de las galerías y subastas, a los 44 restantes. En función de la distribución entre los artistas de las 773 obras compradas, pueden deducirse las prioridades de los museos a la hora de comprar, en principio, con el criterio de llenar huecos o completar la trayectoria de los artistas en los diferentes museos. Para no hacer tedioso el relato, en relación con las variables que se introducirán en nuestro modelo, singularizaremos a los artistas del último deciil en alguna de la distribución de frecuencias de los artistas en cuanto a sus obras en las colecciones -WMUSEUMS- (>20), las compradas -BUYMUSEUMS- $(>20)$, en sus precios medios -MANBUYMUSEUMS $(>70.000 €$ ) o en el costo total (> $1 \mathrm{mi}-$ llón€). El amplio periodo cronológico hasta 2002, último año del que disponemos información, hace necesario actualizar el precio al que se adquirieron las 773 obras consideradas. Por ello, se han deflactado dichos precios, enlazando las series del deflactor implícito del GDP Producto Interior Bruto español a euros constantes de 2010 (Prados de la Escosura, 2003 y De la Fuente, 2016).

En el País Vasco, la falta de tradición artística propia hasta finales del siglo XIX y principios del XX no es debida a la falta de creadores, sino a que muchos de ellos se desplazaron a Madrid, como es el caso de Antonio María de Lekuona o Juan de Barroeta Anguisolea, que pese a no ser estrictamente modernos, pues fallecieron recién comenzado el siglo $\mathrm{XX}$, siguieron las corrientes de la pintura española de la época. Los primeros síntomas de modernización, es decir, los que podemos considerar como protomodernos, provienen de los residentes en la Academia Española en Roma. Entre ellos se encuentran Ignacio Zuloaga, José Echenagusia y los alaveses Fernando de Amárica e Ignacio Díaz de Olano.

La introducción de una primera modernidad en la pintura vasca viene del trabajo de Adolfo Guiard, Anselmo Guinea y Dario Regoyos. Otros artistas trataron de unir tradición y modernidad como Pablo Uranga o Manuel Losada, mientras que Juan
Echevarría, Benito Berrueta, Francisco Iturrino y Ricardo Baroja interpretaron la modernidad desde postulados diferentes. En la primera parte del siglo XX y hasta la Guerra Civil se pone de manifiesto la capacidad para absorber los postulados de la modernidad internacional, produciendo dos tipos de artistas, los que renuevan la tradición artística nacional y los que se inscriben en el nuevo arte moderno, incorporando postulados de la vanguardia internacional. Entre ellos se encuentra el eclecticismo de Antonio Guezala, el simbolismo de Angel Larroque, el expresionismo de Ascensio Martiarena, el fauvismo de Bienabe Artía, la influencia del cubismo en Gustavo de Maeztu y Juan de Aranoa o el surrealismo más poético de José María Ucelay. Paralelamente surge el interés por la identidad territorial al albur de la ideología nacionalista con fines propagandísticos y artistas como los Zubiaurre y Uranga participan de estos postulados. Una excepción es Elías Salaverría, que representa temas costumbristas, desprovistos de todo folklorismo. Pero los más vanguardistas en la interpretación de dichos temas son los hermanos Arrúe, en particular José, Aurelio Arteta o Julián Tellaeche. El introductor de esta modernidad en la escultura vasca fue sin duda Francisco Durrio, aunque no estuvo exenta de localismos en las figuras de Nemesio Mogrobejo, Quintín de la Torre y Moisés Huerta. Durante el primer franquismo se produjo un interés de los artistas por asociarse para cubrir las carencias existentes en el panorama artístico. El camino más común del arte vasco de posguerra hasta los años 50 fue el paisajismo, el retrato y el bodegón, en clave impresionista o con una composición escorada hacia el cubismo. Artistas como Gaspar Montes Iturrioz producen obras de gran calidad, pero desideologizadas en comparación con el trabajo de los artistas que habían colaborado con la República.

En el panorama internacional, mientras Europa se reconstruye de los efectos de la II Guerra Mundial, los centros de actividad y mercado artístico se trasladan a Nueva York, donde se gestarán los principales movimientos de vanguardia. Mientras tanto en el País Vasco se desarrolla un arte figurativo, sin tópicos, pero que permitió a determinados artistas introducir algunos planteamientos de vanguardia y por ello los consideramos ya contemporáneos. Este es el caso de $\mathrm{M}^{\mathrm{a}}$ Paz Jiménez, Menchu Gal o Ignacio Echandi. Sin embargo, curiosamente, fue la construcción de la Basílica de Aranzazu, a iniciativa de los franciscanos, la que supuso la puesta en valor de los escultores vascos de vanguardia, cuyo trabajo era más conocido fuera del territorio nacional, como era el caso de 
Jorge Oteiza -que partiendo de las vanguardias históricas hará una escultura muy intelectualizada con el concepto del vacío como prioritario- y Eduardo Chillida -que trabaja siempre con la escala humana-. Así, si añadimos a los dos anteriores a Agustín Ibarrola y su experimentación permanente y a los escultores en madera Néstor Basterretxea y Remigio Mendiburu, se configura un quinteto de individualidades como referentes plásticos vascos a partir de la década de 1970.

La pintura abstracta de la década de 1960 siguió los postulados del expresionismo y estuvo representada por Rafael Ruiz Balerdi, José Luis Zumeta o Amable Arias, o el informalismo de Gabriel Ramos Uranga. Los artistas de las décadas de 1970 y 1980 son en su mayoría resultado de experiencias individuales, aunque no escapan a las divisiones estilísticas del panorama nacional e internacional y, lógicamente por razón de edad, es necesario rebajar los criterios de cantidad y/o precio de sus obras en las colecciones. En ese grupo se encuentra Antonio Sistiaga o Andrés Nagel. Las propuestas son muy dispares y resulta casi imposible agruparlos por tendencias estilísticas, ya que no existen como tales en el arte actual. Así, a caballo entre la figuración y la abstracción se encuentra el trabajo de Marta Cárdenas o el postsurrealismo de Vicente Ameztoy. En la generación más joven están los representantes del realismo, al que en su día estuvo adscrito Darío Urzay, y que están representados por Clara Gangutia, Jesús Mari Lazkano o Mari Puri Herrero. En la actualidad se encuentran artistas cuyas trayectorias se han consolidado como Juan Luis Goenaga, Alberto Rementeria, José Ramón Amondarain, Alejandro Garmendia, Darío Urzay y Fernando Illana. Hay también un grupo de alaveses cuyo trabajo comienza a ser un punto de referencia museográfico y de creación artística. El grupo está formado por Juan Mieg, Carmelo Ortiz Elguea e Iñaki Cerrajería. La escultura de la década de 1990 está representada por un grupo de artistas de generaciones y planteamientos distintos, en los que resulta evidente la influencia de las realizaciones del decenio anterior, quizá con la salvedad de las instalaciones y montajes que constituyen el trabajo de Txomin Badiola o Juan Luis Moraza. En este contexto también desarrollan su trabajo un grupo de mujeres escultoras muy notable que ha portado individualmente cualidades plásticas importantes y cuyas representantes más importantes son Cristina Iglesias y Dora Salazar.

Como resume la Tabla 1, cronológicamente las obras se compraron fundamentalmente, tanto en número de artistas, 51, como especialmente de obras, 526, tras la muerte del general Franco y especialmente de entre ellos, de 33 durante el periodo de la casi plena capacidad fiscal y económica de la que disfrutan las Diputaciones Forales con el Concierto Económico Vasco en el contexto democrático español desde 1979. La media del precio pagado por las obras de cada artista en euros constantes de 2010 es de 23.400 y la mediana de casi $11.000 €$. Hay 10 artistas con el precio medio de sus obras superiores a $70.000 €$ : cinco modernos, Regoyos -casi $179.000 €$ de precio medio, el pintor español que aunque nació en Asturias en 1857 y se formó académicamente en Madrid, como durante buena parte de su vida adulta, entre 1883 y 1910, tuvo su residencia familiar en el País Vasco hasta poco antes de su muerte en 1913, para la bibliografía vasca se considera "artística y espiritualmente vasco" (Eusko Ikaskuntza, 2018, pág. Regoyos)-, Arteta, Echenagusia, Echeverría y Mogrobejo y cinco contemporáneos, tres de los escultores iconos (Chillida, Ibarrola y Mendiburu) más Larrea y Mieg. Regoyos encabeza también la lista de nuestros 91 pintores por el monto total de euros en adquisiciones con 5.5 millones de euros, seguido por otros cinco artistas con más de un millón -dos de los escultores contemporáneos vascos más conocidos, Chillida y Basterretxea y tres representantes de los vascos "modernos"- Echenagusia, Díaz de Olano y Arteta.

Finalmente, introduciremos en nuestro modelo una variable -ONLINEBIL- de los pintores y sus obras que aparecen en el Catálogo on-line del Museo de Bilbao, intentando captar un cierto "Masterpiece Effect", no tanto con el procedimiento de los trabajos citados en (Asheinfeler \& Graddy, 2003, págs. 770-773), sino en la línea de los que intentan medir el efecto a través del espacio ocupado por los "Masterartists" en otras fuentes (O'Hagan \& Kelly, 2005) (Hellmanzik, 2016), mediante el número de obras en dicho catálogo de 75 de nuestros artistas que allí aparecen con una mediana de 3 obras. Encabezados por un trío de artistas modernos con más de 30 obras -Iturrino, Arteta y Guiard-, otros 13 artistas están representados con 10 o más, entre los que predominan también el resto de modernos más conocidos y con solo cuatro contemporáneos presentes, los tres escultores Oteiza, Chillida y Basterretxea y el pintor Vicente Larrea.

\section{b. El Mercado de las subastas de los artistas vascos, 1985-2017}

El ya citado trabajo de Asheinfeler y Graddy (2003, págs. 764-765) resume brillantemente el funcio- 
namiento de las subastas de arte basado en las dos casas de subastas internacionalmente más conocidas, Sotheby's y Christie's, fundadas en Londres en 1744 y 1766 respectivamente. Los datos ofrecidos por ambas han sido los más utilizados por muchos trabajos, bien directamente de los catálogos de ambas casas, por ejemplo, entre los más conocidos, los ya citados Mei y Moses (2002) (2005), hasta los más recientes (Stepanova, 2017), bien de compilaciones y anuarios que amplían a otras casas importantes de subastas, como el Annual Series Index (Agnello \& Xu, 2008) o el de E. Mayer (Barre, Docclo, \& Ginsburgh, 1991). Pero con la aparición de portales especializados en subastas de arte en la Web desde fines del siglo XX, algunos trabajos empezaron a utilizar esos portales para construir sus bases de datos con muchas más casas de subastas de todo el mundo. Así, (Renneboog \& Van Houtte, 2002) utilizaron ArQuest para su estudio sobre subastas de pintores belgas entre 1970 y 1990; (MacAndrew \& Thompson, 2007) la compilación de Kusin \& Company con datos de ArtNet y ArtFact para el de las subastas de obras de catorce impresionistas entre 1985 y 2001; (Renneboog \& Spaenjers, 2013) utilizaron el Art Sales Index para más de un millón de observaciones sobre 4.490 artistas entre 1957 y 2007; (Galbraith \& Hodgson, 2015) utilizaron ArtPrice para los precios de las subastas de 51 artistas del Rococó y del Neoclasicismo entre 1986 y 2012; (Anderson, Ekelund, Jackson \& Tollison, 2016) usaron los de Askart.com para los de 33 artistas americanos nacidos antes de 1900; y (Hellmanzik, 2016) los de ArtValue entre 1988 y 2007/2010 junto a otra base de datos de grandes exposiciones para estimar la importancia contemporánea de los artistas.

Nuestra segunda base de datos, como la de Galbraith y Hodgson, está construida usando ArtPrice, fundada en 1987, que presume de contener cerca de 30 millones de obras subastadas de más de 650.000 artistas. La información no gratuita que contiene permite conocer, para cada artista, el número de sus obras subastadas (Pinturas, Dibujos y acuarelas, esculturas, grabados y otros objetos) y para cada una de ellas, ordenadas por la fecha de la subasta, el título, la técnica/soporte, las medidas, el precio mínimo y máximo estimado en euros, el resultado del remate y la casa donde se realizó la subasta. Entre agosto y septiembre de 2017, recopilamos la información dis- ponible sobre todas las pinturas y esculturas subastadas desde 1985 -la primera subasta de nuestros 91 artistas es un Zuloaga el 31 de octubre de ese año-, hasta fin de junio de 2017.

La tabla 2 muestra la estadística descriptiva de esta segunda bases de datos agrupando la información sobre 2.092 obras rematadas $-47.5 \%$ de las subastadas- según las características de las subastas, el período cuando esta se realizó, las casas de subastas y su ubicación geográfica -101 internacionales con 434 obras y 26 españolas con los restantes 1.658- y las físicas de las obras (tamaño y material) de las 1.827 pinturas y dibujos y 265 esculturas rematadas de nuestros 91 artistas. Con una mediana de ocho obras rematadas, 15 artistas sobresalen con más de 50, encabezados muy destacadamente por Eduardo Chillida con 195 obras vendidas. Solo los pintores contemporáneos Menchu Gal, Carmelo Barrena e Ignacio García Ergüín, son novedosos en este listado. Por el precio medio de los remates, Chillida es también el destacado, seguido por 16 aristas con precios medios superiores a $9.000 €$, entre los que, salvo los escultores contemporáneos Oteiza y Cristina Iglesias, el pintor/escultor Andrés Nagel y la mencionada Menchu Gal, el resto lo forman los ya mencionados habituales modernos. Como consecuencia combinada, por el total de ventas, de nuevo sobresale Eduardo Chillida, pues los más de 51,5 millones de Euros representan que las obras rematadas del más conocido artista vasco acaparan el $69 \%$ de las ventas conjuntas de los 91 artistas. Muy alejados, otros 11 que vendieron más de $300.000 €$, los modernos Zuloaga, Regollos, los dos hermanos Zubiaurre, Iturrino, Echenagusia y Maeztu y tres contemporáneos ya mencionados, los escultores Jorge Oteiza y Cristina Iglesias y la pintora Menchu Gal. La cronología de las subastas, se ha agrupado en los cuatro últimos periodos económicos según el crecimiento del GDP español (Prados de la Escosura, 2016, pág. 18). El precio -medio y mediano- de las 265 esculturas es aproximadamente 15 veces superior al de las 1.827 pinturas. Ello se explica especialmente, porque entre las 58 obras que alcanzaron un remate superior a los 300.000 euros constantes, todas salvo una subastadas en Christie's y Sotheby's, solamente hay siete pinturas -todas ellas de Zuloaga- y las 41 restantes son esculturas de Eduardo Chillida. Finalmente, las obras pictóricas son mayoritariamente óleos frente a otros procedimientos. 
Tabla 2

BASE DE DATOS DE SUBASTAS DE ARTISTAS VASCOS. ESTADÍSTICA DESCRIPTIVA (EN EUROS CONSTANTES DE 2010)

\begin{tabular}{|c|c|c|c|c|c|c|}
\hline & TOTAL & MIN. & MÁX. & MEDIA & MEDIANA & DESV. EST. \\
\hline Artistas (COD_A) & 91 & & & & & \\
\hline \multicolumn{7}{|l|}{ b.1. Características de las subastas } \\
\hline b.1.1. Total de obras subastadas & 4.401 & 1 & 470 & 48,36 & 16 & 73,87 \\
\hline b.1.2. Obras originales (COD_W) & 2.092 & 1 & 195 & 22,98 & 8 & 34,27 \\
\hline b.1.3. Precio medio de los remates & & 40 & 4.256 .292 & 35.762 & 2.506 & 170.867 \\
\hline b.1.4. Ventas totales por artista & 74.812 .634 & 240 & 51.579 .960 & 822.117 & 23.698 & 51.579 .960 \\
\hline $\begin{array}{l}\text { b.1.4.1. Precio medio de los remates } \\
\text { por artista }\end{array}$ & & 120 & 264.513 & 8.193 & 2.019 & 29.101 \\
\hline \multicolumn{7}{|l|}{ b.1.5. Cronología de los remates } \\
\hline \multicolumn{7}{|l|}{ b.1.5.1. (1985-1992) } \\
\hline b.1.5.1.1. Obras & 123 & 2 & 54 & 15,38 & & \\
\hline b.1.5.1.2. Ventas totales & 5.162 .607 & 159 & 589.871 & 41.972 & 8.433 & 97.368 \\
\hline \multicolumn{7}{|l|}{ b.1.5.2. (1993-2007) } \\
\hline b.1.5.2.1. Obras & 1.046 & 29 & 99 & 69,73 & & \\
\hline b.1.5.2.2. Ventas totales & 36.086 .154 & 65 & 2.787 .124 & 34.499 & 3.380 & 139.951 \\
\hline \multicolumn{7}{|l|}{ b.1.5.3. (2008-2013) } \\
\hline b.1.5.3.1. Obras & 554 & 76 & 133 & 92,17 & & \\
\hline b.1.5.3.2. Ventas totales & 30.146 .674 & 50 & 4.256 .292 & 54.318 & 2.251 & 260.928 \\
\hline \multicolumn{7}{|l|}{ b.1.5.4. (2014-2017) } \\
\hline b.1.5.4.1 Obras & 369 & 99 & 119 & 92,25 & & \\
\hline b.1.5.4.2. Ventas Totales & 3.419 .201 & 40 & 850.435 & 9.266 & 1.002 & 56.600 \\
\hline \multicolumn{7}{|l|}{$\begin{array}{l}\text { b.1.6. Características de las casas } \\
\text { de subastas }\end{array}$} \\
\hline \multicolumn{7}{|l|}{ b.1.6.1. Subastas en España } \\
\hline b.1.6.1.1. Casas de subastas & 26 & & & & & \\
\hline b.1.6.1.2. Obras & 1.658 & 1 & 312 & 63,85 & 14 & 103,11 \\
\hline b.1.6.1.3. Artistas & & 1 & 55 & 17,08 & 8,5 & 17,88 \\
\hline b.1.6.1.4. Ventas Totales & 13.103 .478 & 3.006 & 2.912 .783 & 503.980 & 158.097 & 743.820 \\
\hline \multicolumn{7}{|l|}{ b.1.6.2. Subastas Internacionales } \\
\hline b.1.6.2.1. Casas & 101 & & & & & \\
\hline b.1.6.2.2. Obras & 434 & 1 & 105 & 4,29 & 1 & 14,43 \\
\hline b.1.6.2.3. Artistas & & 1 & 10 & 1,72 & 1 & 1,7 \\
\hline b.1.6.2.4. Ventas totales & 61.711 .158 & 150 & 33.661 .875 & 611.002 & 7.268 & 3.863 .796 \\
\hline b.1.6.3. Christie's & 6 & & & & & \\
\hline b.1.6.3.1. Obras & 147 & 1 & 102 & 24,5 & 9,5 & 35,5 \\
\hline b.1.6.3.2.Ventas totales & 37.281 .121 & 12.024 & 33.661 .875 & 6.213 .520 & 603.092 & 12.305 .026 \\
\hline b.1.6.4. Sotheby's & 5 & & & & & \\
\hline b.1.6.4.1. Obras & 143 & 1 & 105 & 28,6 & 17 & 38,87 \\
\hline b.1.6.4.2. Ventas totales & 23.585 .433 & 4.044 & 19.985 .546 & 4.717 .087 & 968.502 & 7.693 .011 \\
\hline
\end{tabular}




\begin{tabular}{|c|c|c|c|c|c|c|}
\hline & TOTAL & MIN. & MÁX. & MEDIA & MEDIANA & $\begin{array}{c}\text { DESV. } \\
\text { EST. }\end{array}$ \\
\hline \multicolumn{7}{|c|}{ b.1.7. Características de las obras } \\
\hline b.1.7.1. Pinturas y Dibujos & 1.827 & & & & & \\
\hline b.1.7.1.1. Área $\left(\mathrm{cm}^{2}\right)\left(\mathrm{AREAm}{ }^{2}\right)$ & & 31 & 59.792 & 3.593 & 1.716 & 5.553 \\
\hline b.1.7.1.2. Ventas totales & 23.979 .868 & 40 & 1.139 .937 & 13.125 & 2.013 & 50.075 \\
\hline b.1.7.2. Esculturas & 264 & & & & & \\
\hline b.1.7.2.1. Altura $(\mathrm{cm})($ AREAm) & & 0,22 & 800 & 52,58 & 29,5 & 70,71 \\
\hline b.1.7.2.2. Ventas & 50.833 .257 & 50 & 4.256 .292 & 191.824 & 34.171 & 430.469 \\
\hline \multicolumn{7}{|l|}{ b.1.7.3. Materiales } \\
\hline b.1.7.3.1. Óleos (OIL) & 1.043 & & & & & \\
\hline b.1.7.3.1.1. Área $\left(\mathrm{cm}^{2}\right)$ & & 63 & 59.762 & 4.773 & 3.000 & 5.848 \\
\hline b.1.7.3.1.2. Ventas totales & 19.972 .816 & 60 & 1.139 .937 & 19.131 & 3.711 & 64.133 \\
\hline b.1.7.3.2. Acuarelas' ${ }^{1}$ (WATER) & 305 & & & & & \\
\hline b.1.7.3.2.1. Área $\left(\mathrm{cm}^{2}\right)$ & & 96 & 16.060 & 1.494 & 907 & 1.763 \\
\hline b.1.7.3.2.2. Ventas totales & 906.920 & 60 & 50.220 & 2.974 & 1.409 & 5.256 \\
\hline \multicolumn{7}{|l|}{ b.1.7.3.3. Materiales Mixtos ${ }^{2}$} \\
\hline b.1.7.3.3.1. Área $\left(\mathrm{cm}^{2}\right)$ & 100 & 85 & 56.826 & 7.225 & 4.092 & 10.693 \\
\hline b.1.7.3.3.2. Ventas totales & 2.035 .827 & 110 & 255.332 & 20.564 & 3.999 & 36.818 \\
\hline b.1.7.3.4. Dibujos ${ }^{3}$ (DRAWS) & 379 & & & & & \\
\hline b.1.7.3.4.1. Área $\left(\mathrm{cm}^{2}\right)$ & & 31 & 59.792 & 3.293 & 1.440 & 5.248 \\
\hline b.1.7.3.4.2. Ventas totales & 20.037 .434 & 40 & 1.139 .937 & 14.530 & 2.001 & 56.088 \\
\hline
\end{tabular}

Notas: 1. acuarela + gouache + pastel + sinopia + tempera + cera; 2 . Material Mixto + acrílico + collage; 3. Dibujos = carboncillo + rotulador + tinta + lápiz.

\section{La especificación del análisis multinivel empleado}

Las aproximaciones hedónicas a los precios de las subastas de arte cuando tienen como objeto las obras de un colectivo determinado de artistas, para tener en cuenta la influencia de la autoría en el precio, introducen una serie de variables ficticias. Pero como indicaron Merijn Rengers y Olav Velthuis (2002), al incluir todas las variables en el análisis OLS, estos estudios asumen implícitamente que hay tantos datos de artistas como obras de arte en sus bases de datos, es decir, que no hay jerarquías en los datos. El principal argumento de su trabajo es que los precios en el mercado están determinados por características en diferentes niveles o clústeres, obras de arte, artistas y galerías. Utilizando un modelo multinivel consideran que se usa el número de casos correcto para cada nivel de características, con lo que se estiman correctamente los errores estándar y, además, que dichos modelos proporcionan información sobre la varianza de un nivel en relación con la varianza total. Por tanto, posibilita conocer cuánto de la dife- rencia de precio en las obras de arte se debe a las características de las mismas y cuánto a las características de los artistas que las crearon. Más recientemente (Modugno, Cagnone, \& Giannerini, 2015), tratan los datos de las subastas con un análisis de cross.sectionregresión repetido, tomando los ítems de las obras como unidades del nivel-1 y periodos cronológicos como unidades del nivel-2, en su estudio sobre los precios del Arte Tribal. La amplia bibliografía sobre la utilización de los modelos multinivel en las ciencias sociales y médicas ha destacado en concreto que "comparados con las clásicas regresiones, suponen casi siempre una mejora, aunque en diversos grados: para la predicción, los modelos multinivel pueden ser esenciales, útiles para la reducción de los datos y de gran ayuda para la inferencia causal" (Gelman, 2006, pág. 432). Las justificaciones estadísticas para esta opción metodológica pueden encontrarse en (Gelman \& Hill, 2007 y Goldstein, 2010).

Teniendo en cuenta todo esto, para explotar conjuntamente nuestras dos bases de datos, hemos optado, dadas estas supuestas ventajas, por un mo- 
delo con dos niveles. En el nivel-1 las características hedónicas de las obras de arte rematadas. En el nivel-2, las de los artistas que las crearon. La representación algebraica de nuestro modelo, tomando la variable dependiente el logaritmo natural del precio de remate en euros constantes de 2010, es la siguiente:

Modelo 1: Modelo-Base sin poder explicativo:

$$
\begin{aligned}
& \text { LnPRECIOREMATE }=\beta_{0 i j}+u_{0 j}+e_{0 i j} \\
& {\left[u_{0 j}\right] \sim N\left(0, \Omega_{u}\right): \Omega_{u}=\sigma_{u 0}^{2}} \\
& {\left[e_{0 i j}\right] \sim N\left(0, \Omega_{u}\right): \Omega_{e}=\sigma_{e 0}^{2}}
\end{aligned}
$$

Modelo 2 y 3: Modelo de dos niveles con Efectos Fijos:

$$
\begin{aligned}
& \text { LnPRECIOREMATE }=\beta_{0 i j}+A_{i j}+e_{0 i j} \\
& \text { LnPRECIOREMATE }=\beta_{0 i j}+A_{i j}+B_{j}+u_{0 j}+e_{0 i j}
\end{aligned}
$$

Donde $i$ y $j$ indican los dos niveles observados (obras y artistas), $\beta_{0 i j}$ la constante o precio medio y $A$ y $B$ son los vectores de variables explicativas al nivel de obras y al de artistas, $u_{0 j}$ es el efecto del artista $j$, es decir, la varianza entre artistas y $e_{0 i j}$ es el término de error residual conjunto entre artistas y sus obras, es decir, la varianza entre las obras de dentro de cada artista.

El vector $A_{i j}$ común en ambos modelos, engloba las siguientes variables de las obras subastadas: variables ficticias para SCULPture Escultura (otras $=0$ ); también variables ficticias para el material de las pinturas como OILÓleo, WATERAcuarela y DRAW Dibujo (Materiales Mixtos=0); AÁREA, en $\mathrm{m}^{2}$ para las pinturas (alto $x$ ancho) y en metros de altura para las esculturas, estandarizada para hacer las esculturas comparables con las obras de arte de dos dimensiones; dos variables ficticias para el lugar de celebración de la subasta, INTERNATC (En España=0) y CHSOET para las celebradas en las diversas sedes, incluidas las españolas, de Christie's y Sotheby's (Otras $=0$ ); unas variable ficticias para cada uno de los periodos cronológicos en que se celebraron las subastas, 93-07, 08-13 y 14-17 (1985-1992=0) y, finalmente, otra ficticia, DEATH, Fallecido para las obras de artistas fallecidos antes de la subasta (Vivos=0).

Y el vector $B_{j}$, exclusivo del Modelo 3, contiene las variables en el nivel 2 de los artistas, fundamentalmente de nuestra base de datos de los Museos: una ficticia, MODERNModerno, que toma el valor de 1 para los 47 artistas modernos y 0 para los 44 contemporáneos; AUCTSSubastadas, para el número de obras subastadas, incluidas las no rematadas, de cada uno de ellos; WMUSObmuseos, para sus obras en las colecciones de los museos; WBUYMUSOcompradas, para las adquiridas; LnMEANBUYMUSLnMediacompras, el logaritmo natural del precio medio de adquisición en euros constantes de 2010 y, finalmente GUIDBILGibil, con el número de obras reproducidas en la guía on-line del Museo de Bellas Artes de Bilbao.

Como ya habíamos señalado la gran desproporción en el precio de las 365 esculturas sobre la mayoría de las pinturas, especialmente por la incidencia de los altísimos precios de remate de las esculturas de Eduardo Chillida, hemos aplicado el mismo modelo exclusivamente a las 1.827 obras pictóricas, suprimiendo, como es lógico, la variable ficticia SCULP, para testar si su inclusión modificaba sustancialmente los resultados. El software estadístico utilizado es MLwiN del Center for Multilevel Modelling de la Universidad de Bristol (2017).

\section{Resultados y discusión}

En la Tabla 3 hemos traslado el output de las tres ecuaciones mencionadas de nuestro modelo de dos niveles, primero las referidas a todas las obras artísticas y sus respectivos creadores y, en segundo lugar, las referidas exclusivamente a las obras bidimensionales y sus artistas. Comenzaremos con una evaluación general de los modelos. La línea final de la tabla 3 muestra la desviación estadística, calculada como menos dos veces la probabilidad logarítmica ("log-likelihood"). La diferencia de desviaciones entre dos modelos sucesivos proporciona el test de probabilidad estadística para comparar el grado de adecuación de los dos modelos. La desviación de un modelo simple sin efectos de los artistas (no mostrado en la tabla) es $D=8.884,808$, mientras que en nuestro modelo 1 es de 7.563,773. La prueba estadística LR (diferencia entre desviaciones) y su valor-p para testar cuál de los dos modelos es preferible es: $\chi_{1}^{2}=$ $1.321,035, p<0.001$. Como el último valor es cero, por tanto, el modelo de dos niveles se ajusta mejor a los datos que un modelo de nivel único, por lo que podemos concluir que las 2.098 obras no actúan como 2.092 observaciones independientes, si no mejor distribuidas entre sus respectivos creadores. Igualmente, el valor $\mathrm{p}<0.001$ del estadístico LR entre el modelo 1 y el modelo 2 (1.699,68 para todas las obras y $1.530,91$ solo para las bidimensionales), y entre el Modelo 2 y el Modelo 3 $(83,83$ y 69,28$)$ confirman que los predictores adicionales mejoran significativamente el ajuste de los modelos. Los coeficientes de partición de la varianza (VPC), también llamados coeficiente de correlación interclase (ICC), informan de la proporción de 
la varianza observada en los residuos que se puede suponer por las diferencias entre los grupos y los individuos, ya que es una media de la semejanza de los individuos en el grupo del nivel inferior y la diferencia en un marco más global. Esos coeficientes permiten, por tanto, suponer la relativa importancia de los artistas y las obras como origen de la varianza de los precios de estas últimas. Como informa la Tabla 3, en el Modelo 1 básico con todas las obras, el 3\% de la varianza de los residuos podría ser explicada por los niveles jerarquizados -el $2,7 \%$ en las pinturas y dibujos- en donde $34 \%$ de la varianza de los precios podría explicarse entre los artistas y el $66 \%$ lo haría, dentro de los artistas, entre sus obras. Si se incluyen las variables relacionadas con las características de las obras, como en el Modelo 2 el reparto es $54 \%$ de los artistas y $46 \%$ entre sus obras. Incluyendo las variables relacionadas con los artistas en el Modelo 3, se vuelve a lo valores del modelo 1,34 y $66 \%$. En los modelos referidos solamente a pinturas y dibujos, en el Modelo 1 los valores son $29 \%$ y $71 \%$ para artistas y obras respectivamente. Al incluir las características de las obras, el reparto es de $45 \%$ y $55 \%$, volviendo a los valores conocidos de $34 \%$ y $66 \%$ cuando se incluyen las de los artistas. Estos resultados parecen indicar que en el caso de las obras bidimensionales, sus características propias explican mejor la variación de los precios que las características del autor de las mismas, aunque finalmente, en el Modelo 3, con las VPCs de ambos niveles, la proporción sea la misma con o sin inclusión de las esculturas.

Para no hacer parsimoniosa la presentación de los coeficientes obtenidos, nos referiremos exclusivamente a los del Modelo 3, reseñando, en primer lugar, los coeficientes de las características de las obras de arte que conforman el Modelo 2. De los 11 coeficientes, 9 son significativos al $99 \%$ y 2 al $95 \%$, aunque el referido al del artista ya fallecido en el año de la subasta lo sea solo al $90 \%$ en el modelo exclusivo de pinturas.

Si recordamos que de las 2.092 obras de nuestra base de datos, el $94 \%$ fueron rematadas en subastas celebradas en España, es de suponer que la coyuntura cíclica de la economía española tenga un importante impacto en los niveles de precios de un bien duradero, de inversión o consumo, como las obras de arte. Por ello, como ya indicaban las medianas de los precios en los periodos en que la literatura económica periodiza el crecimiento del GDP español, los coeficientes obtenidos ratifican que tras 1985-1992, el primer ci- clo expansivo de la economía española tras la restauración democrática, los tres subsiguientes inciden, de manera crecientemente negativa en los precios. Si el impacto en el precio de cada variable hedónica puede aproximarse mediante un cálculo que consiste en tomar el coeficiente del exponente y sustraerle 1 (100 x e eoef-1), los impactos sobre el precio medio en los tres subsiguientes periodos a $1985-1992$ son de $-18 \%$, $-48 \%$ y $-66 \%$ respectivamente, como vemos, especialmente fuertes en los dos últimos periodos, desde 2008, con la Gran Recesión de la economía mundial, más extensa e intensa en la economía española.

Como indican los más recientes trabajos sobre los determinantes de los precios en el mercado secundario o terciario de las obras de arte realizadas por artistas seleccionados por región o estilo artístico, en los que se citan muchos trabajos anteriores, las características físicas de las obras, el fallecimiento del artista antes de la venta y la casa de subasta son un buen predictor del nivel del precio (Rengers \& Velthuis, 2002), (Higgs \& Forster, 2014), (Galbraith \& Hodgson, 2015), (Anderson, Ekelund, Jackson, \& Tollison, 2016) y (Farrell, Fry \& Fry, 2017). Por ello, también en las obras de los artistas vascos, todos los coeficientes son significativos al $99 \%$ y con los signos esperados. Así, un aumento de tamaño de una desviación estándar en la superficie (o altura en las esculturas), $0.58 \mathrm{~m}^{2}$, repercute positivamente en el $57 \%$ del precio medio de los remates. Igualmente, como era de esperar, las esculturas y los óleos aumentan significativamente el precio medio (131\% y $138 \%)$, mientras que las acuarelas y dibujos lo disminuyen también muy significativamente $(-34 \%$ y $-73 \%$ ). El gran predominio de obras subastadas en España probablemente incida en que las subastas en el extranjero tengan un coeficiente negativo, con un impacto en el precio del $-17 \%$ lo que difiere con un coeficiente negativo equivalente para las casas de subastas australianas en el trabajo citado de Helen Higgs y John Forster (2014), mientras que se ratifica también para las obras vascas, el impacto positivo en el precio medio si la obra se subasta en Sotheby's o Christie's (168\% para el conjunto y $148 \%$ para las pinturas). Finalmente, en este set de características, el fallecimiento del artista antes de la fecha de la subasta, también en el caso vasco incide muy positivamente en el precio $(58 \%$ en el total de las obras y $35 \%$ en las pinturas).

Finalizaremos esta presentación de resultados con los seis coeficientes referidos a las características 
de los artistas. En tres de estos coeficientes no podemos descartar la $H_{0}$ de que son distintos de cero. No es significativa la incidencia (y signo negativo) en el precio de los remates de la variable proxi sobre el carácter moderno o contemporáneo de los autores. Y tampoco, dos de nuestras variables de la base de datos de Museos. En contra de lo esperado, ni la cantidad de obras de cada artista en las colecciones vascas, ni el número de esas obras que ingresaron por adquisición institucional, son significativas. Por tanto, no puede confirmarse, al menos con la formulación concreta que hemos dado a esas variables, la hipótesis asumida por la sociología del arte sobre la incidencia en la valoración económica directa de la "visibilidad" proporcionada a las obras de los artistas en los museos (Moulin, R.; Passeron, J-D; Pasquier, D.; Porto-Vasquez, F., 1984). La relativamente pequeña dimensión de estos museos vascos frente a otros grandes museos y los reducidos tamaños de la exposición de sus colecciones permanentes podrían explicar la no significatividad de nuestras dos variables. ${ }^{3}$

Sin embargo, otras dos variables que inciden también en la visibilidad, bien para públicos más amplios que los del más visitado físicamente de los museos vascos, descontando el Guggenheim de Bilbao, como los visitantes virtuales del Bellas Artes de la capital vizcaína, o para el público interesado en las subastas, son significativos a más de $95 \%$. Como ya hemos comentado, ambos coeficientes de las variables OSUBASTADAS y GUIBIL tienen una lectura similar a la de los trabajos que miden el efecto del espacio físico ocupado por los artistas en otras fuentes como indicador de su reputación artística. Por ejemplo (Renneboog \& Spaenjers, 2013), utilizan una "TextBook dummy" para la inclusión de un artista en la obra de Gardner y otras para la presencia en la Documenta de Kassel (Campos \& Leite Barbosa, 2009); incluyen dummy variables sobre la inclusión de la obra de un artista latinoamericano en un Catalogue raisonné, libro de arte o expuesta previamente, se- gún los catálogos de Sotheby's de Nueva York; y (Hellmanzik, 2016) como ya hemos comentado, utiliza el espacio dedicado a cada artista en términos de columnas y pulgadas en dos diccionarios de artistas y su aparición en una historia de Salones y Exposiciones Las obras de cada artista reproducidas en la Guía on-line y banco de imágenes del museo bilbaíno ${ }^{4}$ son hipervínculos a otras tantas páginas completas con información detallada tanto de la obra como del autor. Igualmente, cada obra de arte citada en la base de datos de pago de ArtPrice lleva a una página con información de autor y a otra con los datos de la obra subastada ofrecidos por el catálogo de la casa de subastas. Por tanto, es de esperar que una mayor presencia del artista -en número de páginas específicas- en ambas fuentes, tenga un impacto positivo en los precios de remate de obras de ese artista por significar una mayor "visibilidad" para los visitantes virtuales del Museo de Bilbao y para los aficionados y coleccionistas consultores de los catálogos de subastas. Por cada obra subastada el impacto positivo en el precio es del $0.5 \%$ y por cada obra en la Guía online del Museo de Bilbao el impacto es del $3.15 \%$ y $2.74 \%$ para el total de obras y las pinturas respectivamente.

Y finalmente llegamos a los resultados de la variable que justifica fundamentalmente nuestro trabajo según la agenda de investigación sugerida en 1997 por (Pommerehne \& Feld), sobre el impacto que el precio medio de adquisición de las obras de un pintor por las colecciones públicas vascas entre 1912 y 2002 pudieran tener en las subastas de obras de esos mismos artistas entre 1985 y 2017. Los resultados del coeficiente en el Modelo 3, (0,306 y 0,371, ambos significativos al $99 \%$ ) significan que el impacto positivo de la media del precio museístico de las obras adquiridas de un autor en el precio medio de los remates de las obras del mismo, es del $36 \%$ en el conjunto y hasta del $45 \%$ para las obras bidimensionales.

\footnotetext{
3 Para dar un idea del tamaño de los museos vascos, frente a 1.169.404 visitantes del Guggenheim de Bilbao en 2016 (Guggenheim Bilbao, 2016, pág. 34), el BBAA de Bilbao recibió 269.977 visitantes el mismo año (Museo de Bellas Artes de Bilbao, 2017, pág. 77), el Artium de Vitoria fue visitado en 2017 por 94.911 personas (http://www.artium.org/es/explora/sala-de-prensa/noticias/item/60621-los-visitantes-de-artium-crecieron-un-nueve-por-ciento-en-2017-hasta-rozar-los-95-000), mientras que el BeIlas Artes de Vitoria solo recibio 22,166 visitantes en 2017. (http://www.europapress.es/euskadi/noticia-afluencia-publicomuseos-alava-aumenta-22-2017-20180110112413.html).

${ }^{4}$ Accesible en https://www.museobilbao.com/coleccion-online.php. La página de cada artista mencionado consta de hipervínculos en las miniaturas de sus obras (10 por página), que llevan a una página completa de descripción de cada obra e información biográfica del autor en la base de datos ARTEDER.
} 


\section{Conclusiones}

Para analizar los determinantes hedónicos de los precios de remate en las subastas de obras creadas por artistas vascos modernos y contemporáneos hemos utilizado conjuntamente dos bases de datos de distinta procedencia. Por una parte, la de los inventarios de los cuatro museos públicos de las tres capitales vascas más los de las Diputaciones Forales de Vizcaya y Guipúzcoa. Estas instituciones habían adquirido a lo largo del siglo XX y hasta 2002, al menos tres obras de 91 artistas vascos de los siglos XIX y XX, 773 obras en total, hasta alcanzar en 2002, sumando donaciones y préstamos, 1.262 esculturas, pinturas y dibujos. Con esta información hemos podido construir unas novedosas variables para testar si estas compras públicas tuvieron algún impacto en el precio de las obras subastadas. Para esto último, se construyó una segunda base de datos, habitual en la literatura sobre el precio de las obras de arte en el mercado terciario, con la información contenida en ArtPrice de 4.401 obras subastadas -2.092 rematadas- de esos mismos 91 artistas entre 1985 y 2017.

Con los datos combinados de ambas bases se ha construido un modelo de dos niveles que estadísticamente parece preferible al modelo hedónico simple habitual. Los Variance Partition Coefficients (VPC) nos permiten establecer la importancia relativa del artista y las obras como fuentes de variación de los precios de las obras. Como se ha visto en la Sección 4, del 3\% de la varianza no explicada, el 34\% parece poder atribuirse a las diferencias entre artistas y un $66 \%$ dentro de un artista a las características de sus obras ( $29 \%$ y $71 \%$ en el modelo exclusivo de pinturas y dibujos). Los 17 predictores adicionales empleados mejoran significativamente los modelos diseñados, de acuerdo con las diferencias observadas en las desviaciones.

Se han utilizado 11 coeficientes de características de las obras rematadas. Con ellos hemos medido el impacto de la coyuntura económica española, negativa en los precios de los remates tras 1992 y, coincidiendo con la literatura, los impactos positivos del aumento del tamaño de la obra; del fallecimiento de artista antes del año de la subasta; de las esculturas sobre las bidimensionales y en estas últimas, de los óleos sobre el resto de procedimientos; y, aunque la realización de la subasta en España tiene un efecto positivo inesperado, se confirma plenamente el fuerte impacto positivo de las subastas de Christie's y Sotheby's.
Igualmente, de los seis coeficientes de las características específicas de los artistas, aunque no tiene significación estadística el impacto en los precios las obras de cada artista en las colecciones públicas vascas, ni el número de las que adquirieron de cada uno de ellos, ni la distinción entre modernos y contemporáneos, sí son muy significativos los impactos de los otros tres. Dos se refieren a la reputación o reconocimiento de los artistas vascos mediante el número de obras subastadas y el de obras incluidas en la Guía online del Museo de BeIlas Artes de Bilbao.

Finalmente, al establecer que el precio medio de adquisición de obras de artistas vascos por los museos y diputaciones tiene un impacto positivo en el precio medio de remate de entre $36 \%$ y el $45 \%$, creemos que queda plenamente demostrada la hipótesis principal de nuestro ejercicio. El precio de adquisición de obras de arte por las instituciones públicas y museos vascos, además de incrementar las obras de los artistas en sus colecciones, parece producir un efecto positivo muy significativo en las subastas de obras de los artistas vascos.

\section{Bibliografía}

AGNELLO, R.; XU, X. (2008). Price for paintigs by African American Artitis and their comtemporaries: Does race matter? Obtenido de Working Pappers Series, Department of Economics, Algred Lerner Colleg Of Business \& Economics, University of Delaware: http://lerner.udel.edu/economics/workingpaper.htm

ALONSO OLEA, E. (1995). El concierto económico (18781937): orígenes y formación de un derecho histórico. Bilbao: Universidad del País Vasco.

ANDERSON, S. C.; EKELUND, R. B.; JACKSON, J. D.; TOLLISON, R. D. (2016). Investiment in early American art: the impact of transaction cost and no-sales returns. Journal of Cultural Economics, 40, 335-357.

ANTIGÜEDAD DEL CASTILLO-OLIVARES, M. D. (1998). EI Museo de la Trinidad, germen del museo público en España. Espacio, Tiempo y Forma. Serie VII. Historia del Arte, 11, 367-396.

ASHENFELTER, O.; GRADDY, K. (2003). Auctions and the Price of Art. Journal of Economic Literature, 41(3), 763-787.

ASHENFELTER, O.; GRADDY, K. (2002). Art Auctions: A Survey of Empirical Studies. Recuperado el 16 de 11 de 2017, de National Bureau of Economic Research (NBER). Working Paper No. 8897: http://www.nber. org/papers/w8997

ASHENFELTER, O.; GRADDY, K. (2010). Art Auction . Recuperado el 16 de 11 de 2017, de CEPS Working Paper No. 203: https://www.princeton.edu/ceps/working papers/203ashenfelter.pdf

BADÍA LACALLE, J. (Comp.). (1975). El concierto económico con Álava y su legislación complementaria. Vitoria: Diputación Foral de Álava. 
BARRE, M. d.; DOCCLO, S.; GINSBURGH, V. (1991). Returns of Impressionist, Modern and Contemporary European Paintings, 1962-1991. Annales d'Economie et de Statistique(35), 143-181.

BEGOÑA, A. d.; BERIAIN, M. J.; MARTÍNEZ DE SALINAS, F. (1982). Museo de Bellas Artes de Álava. Vitoria: Diputación Foral de Álava.

CAMPOS, N. F.; LEITE BARBOSA, R. (2009). Painting and numbers: An economietric investigation of sales, rates prices and return in Latin American art auctions. $O x$ ford Economc Pappers. New Series, 61(1), 28-51.

CANTOR, J. E. (1991). The Museum's Collection. En M. FELDSTEIN, The Economics of Art Museum (págs. 1723). Chicago and London: The University of Chicago Press.

CASTAÑER LÓPEZ, X.; HERNÁNDEZ MARCO, J. L. (2015). A determinant of creativity: the public art acquisitions at the Spanish Basque Country in the 20th Century (Paper presented at XVIIth World Economic History Congress. Kyoto, 2015): https://www.academia. edu/12155 292/A_determinant_of_creativity_the_public_art_acquisitions_at_the_Spanish_Basque_Country_in_the 20th_century

CASTELLS, L. (1987). Modernización y dinámica política de la sociedad guipuzcoana de la Restauración, 18761915. Madrid: Siglo XXI y Universidad del País Vasco.

CHARLTON, C.; RASBASH, J.; BROWNE, W.; HEALY, M.; CAMERON, B. (2017). MLwiN Version 3.00. Obtenido de University of Bristol. Centre of Multilevel Modelling: http://www.bristol.ac.uk/cmm/media/software/ mlwin/downloads/manuals/3-01/manual-web.pdf

DE LA FUENTE, Á. (21 de 01 de 2016). Series enlazadas de PIB y otros agregados de Contabilidad Nacional para España, 1955-2014. Obtenido de Fedea.net - Documentos de macroeconomía: http://documentos.fedea.net/pubs/eee/eee2016-02.pdf

DELOITTE LUXEMBOURG \& ARTTACTIC (2017). Art \& Finance Report 2016. 4th edition. Retrieved 18/11/ 2017: https://www2.deloitte.com/content/dam/Deloitte/ global/Documents/Finance/gx-fsi-art-finance-report2016.pdf

EUSKO IKASKUNTZA (2018). Auñamendi Eusko Entziklopedia.http://aunamendi.eusko-ikaskuntza.eus/

FARRELL, L.; FRY, J. M.; FRY, T. R. (7/11/2017). Determinants of sales and price in auction for three Australian Indigenous artits: to pool or no to pool? Journal of Cultural Economics.

FELDSTEIN, M. (. (1991). The Economics of Art Museums. Chicago: Chicago University Press and NBER Conference Repport.

FREI, B. S.; MEIER, S. (2006). The Economic of Museums. En: V. A. GINSBURGH; D. THROSBY, Handbook of the Economics of Art and Culture. Amsterdam: North Holland - Elsevier, vol. 1, pp. 1017-1050.

FREI, B.; POMMEREHNE, W. W. (1989). Muses and Markets: Explorations in the Economics of the Arts. Oxford: Blackwell.

GALBRAITH, J. W.; HODGSON, D. J. (2015). Innovation, experience and artits' age-valation profiles: evidence from eighteenth-century rococo and neoclassical painters. Journal of Cultural Economics, 39, 259-275.

GALL, L. (8/11/2016). Sotheby's Buys Mei Moses Indices. Obtenido de News of the art world. ArViatic's cultural magazine: http://www.newsoftheartworld.com/ sothebys-buys-mei-moses-index/?lang=en

GARCÍA ZÚÑIGA, M. (2009). El desarrollismo antes del desarrollismo: la industrialización de Álava. Revista de Historia Industrial(41), 91-124.
GASSLER, R. S.; GRACE, R. (1980). The Economic Functions on Non Profite Entrprise: the Case of Art useums. Journal of Cultural Economics, 4(1), 19-32.

GELMAN, A. (2006). Multilevel (Hierarchical) Modeling:What It Can and Cannot Do. Thecnometrics, 48(3), 432-435.

GELMAN, A.; HILL, J. (2007). Data Analysis Using Regression and Multilevel/Hierarchical Models. New York: Cambidge University Press.

GOLDSTEIN, H. (2010). Multilevel Statistical Models, 4th Edition. Hoboken, New York: John Willey \& Sons.

GONZÁLEZ DE ASPURU, S.; SANCRISTÓVAL, P. (2001). El Museo de Bellas Artes de Álavay su colección de Arte Vasco. En: S. GONZÁLEZ DE ASPURU; A. DABOUZA (Eds.), Arte vasco hasta los 50 en el Museo de Bellas Artes de Álava. Vitoria-Gasteiz: Diputación Foral. Servicio de Publicaciones, pp. 19-32.

GORDON, R. J. (1990). The Measurement of Durable Goods Prices (A National Bureau of Economic Research monograph). Chicago: The University ofChicago Press.

GRAMPP, W. D. (1991). Arte, inversión y mecenazgo. Un análisis económico del mercado del arte (original title: Pricing the Priceless). Barcelona: Ariel.

GRAMPP, W. D. (2007). A Colloquy about Art Museum: Economics engages Museology. En: R. Towse, Recent Developments in Cultural Economics. Cheltenham abd Northampton: Edward Elgar, pp. 221-268.

GUGGENHEIM BILBAO (2016). Memoria de actividads 2016. Recuperado el 26/02/2018, de Guggenheim Bilbao Corporativo: http://www.guggenheim-bilbaocorp.eus/wp-content/uploads/2011/03/MEMORIA2016.pdf

HELLMANZIK, C. (2016). Historic art exhibitions and modern - day auction results. Research in Economics, 70, 421-430.

HIGGS, H.; FORSTER, J. (2014). The auction mrket for artworks and their physical dimension: Australia 1986 to 2009. Journal of Cultural Economics, 38, 85104.

JOHNSON, P.; THOMAS, B. (1998). The Economics of Museum: A research Perspective. Journal of Cultural Economics, 22(2), 75-85.

JYRÄMÄ, A. (2008). Identifying organitazional fields in art market - an institutional approach. En: L. Uusitalo, Museum and visual art markets. Helsinki: Helsinki Sochool of Economics, p. 91-110.

KORTEWB, A. G.; KRÄUSSL, R.; VERWIJMEREN, P. (2016). Does It Pay to Invest in Art? A Selection-Corrected Returns Perspective. Review of Financial Studies, 29(4), Available at SSRN: https://dx.doi.org/10.2139/ssrn.2280099.

LUNA, J. J. (1989). El Museo de Bellas Artes de Bilbao. Historia y actualidad. En: Catálogo. Tesoros del Museo de Bellas Artes de Bilbao. Pintura: 1400-1939. Bilbao: Diputación Foral de Vizcaya y Ayuntamiento de Bilbao, pp. 17-20.

MACANDREW, C.; THOMPSON, R. (2007). The collateral value of fine art. Journal of Banking \& Finance, 31, 589-607.

MCANDREW, C. (2017). El mercado español del arte en 2017. Recuperado el 10 de enero de 2018, de Obra Social La Caixa. Arte y Mecenazgo: https://obrasociaIlacaixa.org/documents/10280/666266/05_aym_elmercadoespanoldelarteen2017_es.pdf

MEI, J.; MOSES, M. (2002). Art as an Investment and the Underperformance of Masterpieces. American Econo- 
mic Review, 92(5), 1656-1668. Obtenido de citeseerx.ist. psu.edu/viewdoc/download?doi=10.1.1.210.

MEI, J.; MOSES, M. (2005). Vested Interest and Biased Price Estimates: Evidence from an Auction Market. The Jounal of Finance, 60(5), 2109-2135.

MODUGNO, L.; CAGNONE, S.; GIANNERINI, S. (2015). The Analysis of Tribal Art Prices: a Multilevel Model with Autorregressive Components. Jounal of Applied Statistics, 42(10), 2141-2158.

MORGAN STANLEY (s.f.). Art as an Asset - Morgan Stanley. Recuperado el 18/11/2017, de https://pwm.morganstanley.com/frontstreetgroup/mediahandler/media/56206/Art\%20as\%20an\%20Asset.pdf

MOULIN, R.; PASSERON, J-D; PASQUIER, D.; PORTO-VASQUEZ, F. (1984). Les artistes : Essai de morphologie sociale. Paris: Centre de Sociologie des Arts; EHESS; CNRS.

MUSEO DE BELLAS ARTES DE BILBAO (2017). Cuentas anuales y Memoria 2016. Recuperado el 26 de 02 de 2018, de 110 años. Museo de Bellas Artes de Bilbao: https://www.museobilbao.com/pdf/Resumencuentas2016_cast.pdf

NICOLAU, A.; ZULAIKA, R. (2005). Donostia:el Patrimonio es futuro. Refundación del Museo San Telomo y Anteproyecto para el sector del patrimonio cultural de Donostia-San Sebstial. Recuperado el 12/05/2010, de http://www.donostiakultura.com/

O'HAGAN, J. W. (1998). Art Musems: Collections, Deaccessioning and Donations. Journal of Cultural Economics, 22(2), 197-207.

O'HAGAN, J.; KELLY, E. (2005). Identifying and ranking the most important artits in historical context: methods used and initial results. Hitorical Methods., 38, 118-125.

POGREIN, R. (27/02/2017). Sotheby's Sales Up in Fourth Quarter but Down for the Year. The New York Times, págs. https://www.nytimes.com/2017/02/27/arts/design/ sothebys-sales-up-in-fourth-quarter-but-down-forthe-year.html.

POMMEREHNE, W. W.; FELD, L. P. (1997). The Impact of Museum Purchase on the Auction Prices of Paintings. Journal of Cultural Economics, 21(3), 249-271.
PRADOS DE LA ESCOSURA, L. (2003). El progreso econonómico de España (1850-2000). Madrid: Fundación BBVA.

PRADOS DE LA ESCOSURA, L. (09/2016). Spain's Historical Nastional Accounts: Expenditure and Output, 1850-2015. Recuperado el 4/12/2017, de EHES Working Papers in Economic History: http://www.ehes.org/ EHES_103.pdf

RENGERS, M.; VELTHUIS, O. (2002). Determinants of Prices for Contemporary Art in Dutch Galleries, 19921998. Journal of Cultural Economics, 26(1), 1-28.

RENNEBOOG, L.; SPAENJERS, C. (2013). Buying Beauty: On Prices and Returns in the Art Market. Management Science, 59(1), 36-53.

RENNEBOOG, L.; VAN HOUTTE, T. (2002). The monetary appreciation of painting: from realisme to Magritte. Cambridge Journal of Economics, 26, 331-357.

RIVERA BLANCO, A. (1992). La ciudad levítica. Continuidad y cambio en una ciudad del interior (Vitoria, 1876-1936). Vitoria: Diputación Foral de Álava.

SINGER, L.; LYNCH, G. (1994). Public Choice in the Tertiary Art Market. Journal of Cultural Economics, 18, $119-216$

STEPANOVA, E. (2017). The impact of colour palettes on the price of paintings. Recuperado el 20/01/2018, de Institute of Economics - Scuola Superiore Santa Ana: http://www.lem.sssup.it/WPLem/2017-25.html

TURRELL, B. (1991). The Museum Collection. En: M. Feldstein, The Economic of Art Museum. Chicago: Chicago University Press and NBER Conference Repport, pp. 16-17.

UUSITALO, LIISA (dir.) (2008). Museum and Visual Arts Markets. Helsinki: Helsinki School of Economics.

VICARIO Y PEÑA, N.; ALONSO OLEA, E. (1997). Los conciertos económicos de las provincias vascongadas. Bilbao: Diputación de Bizkaia.

ZUGAZA MIRANDA, M. (1999). Pasado y presente del Museo de Bellas Artes de Bilbao. En: M. ZUGAZA (dir.), Museo de Bellas Artes de Bilbao. Maestros Antiguos y Modernos. Bilbao: Fundación B.B.K., pp. 15-32. 


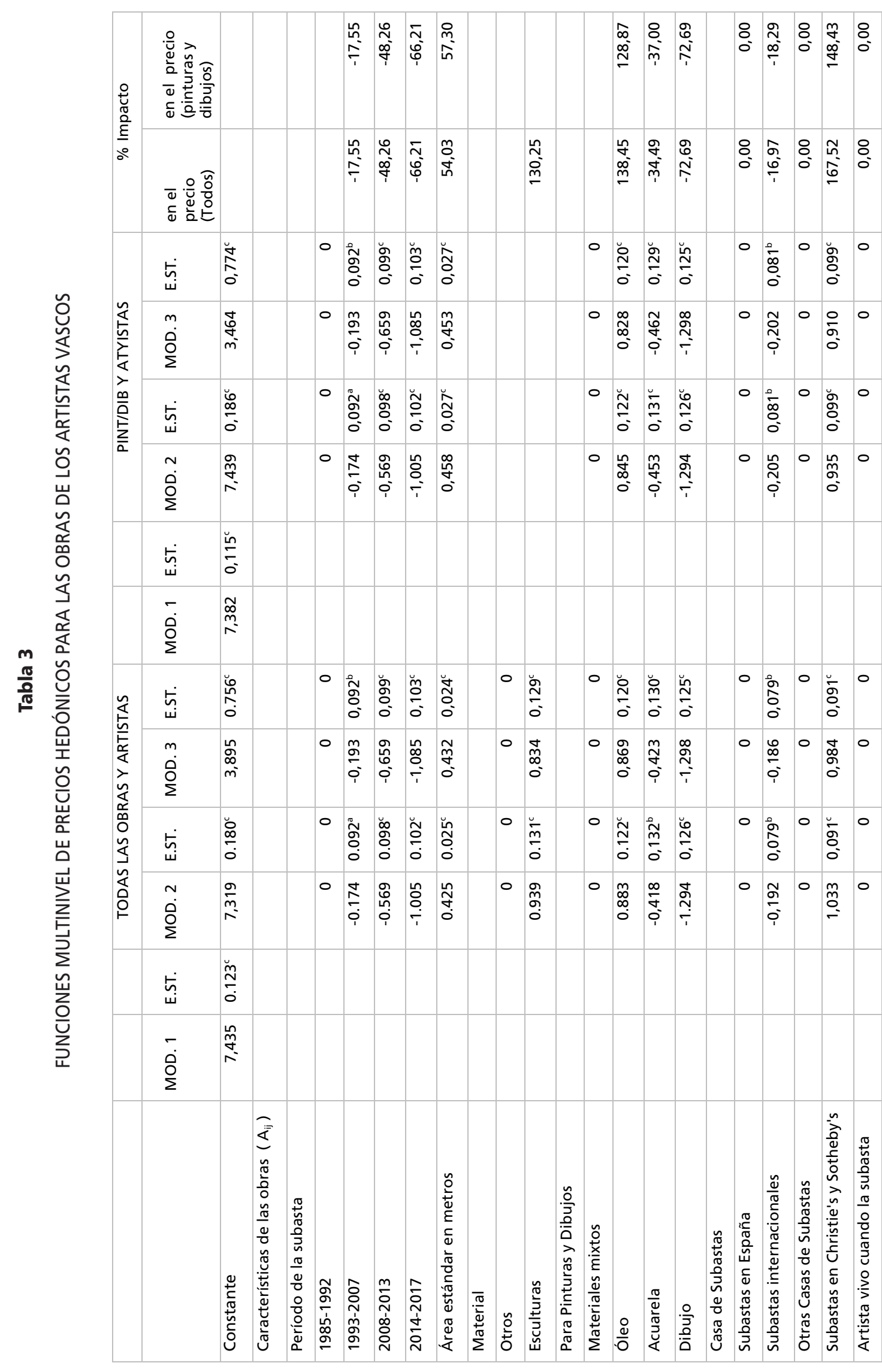




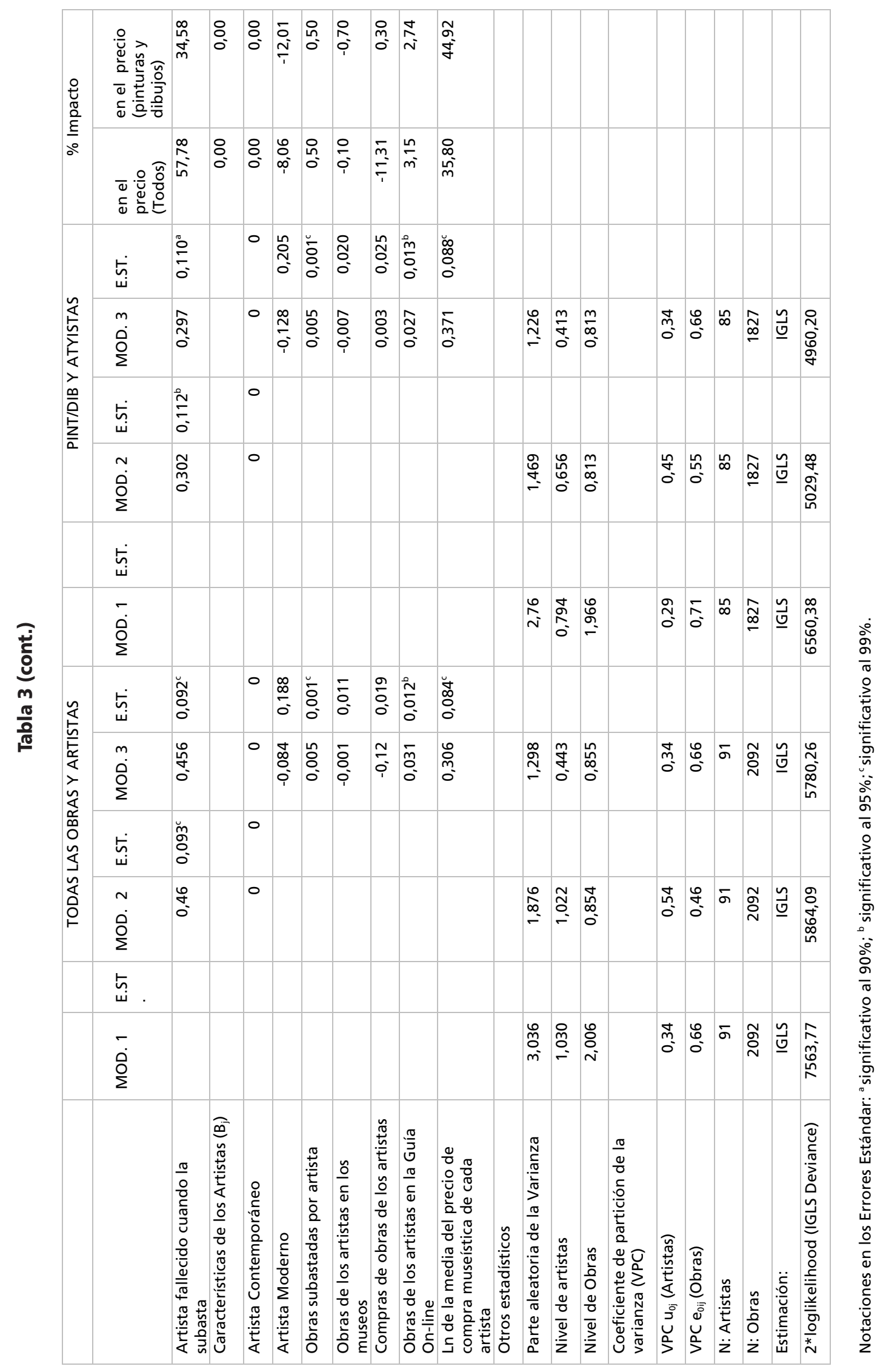




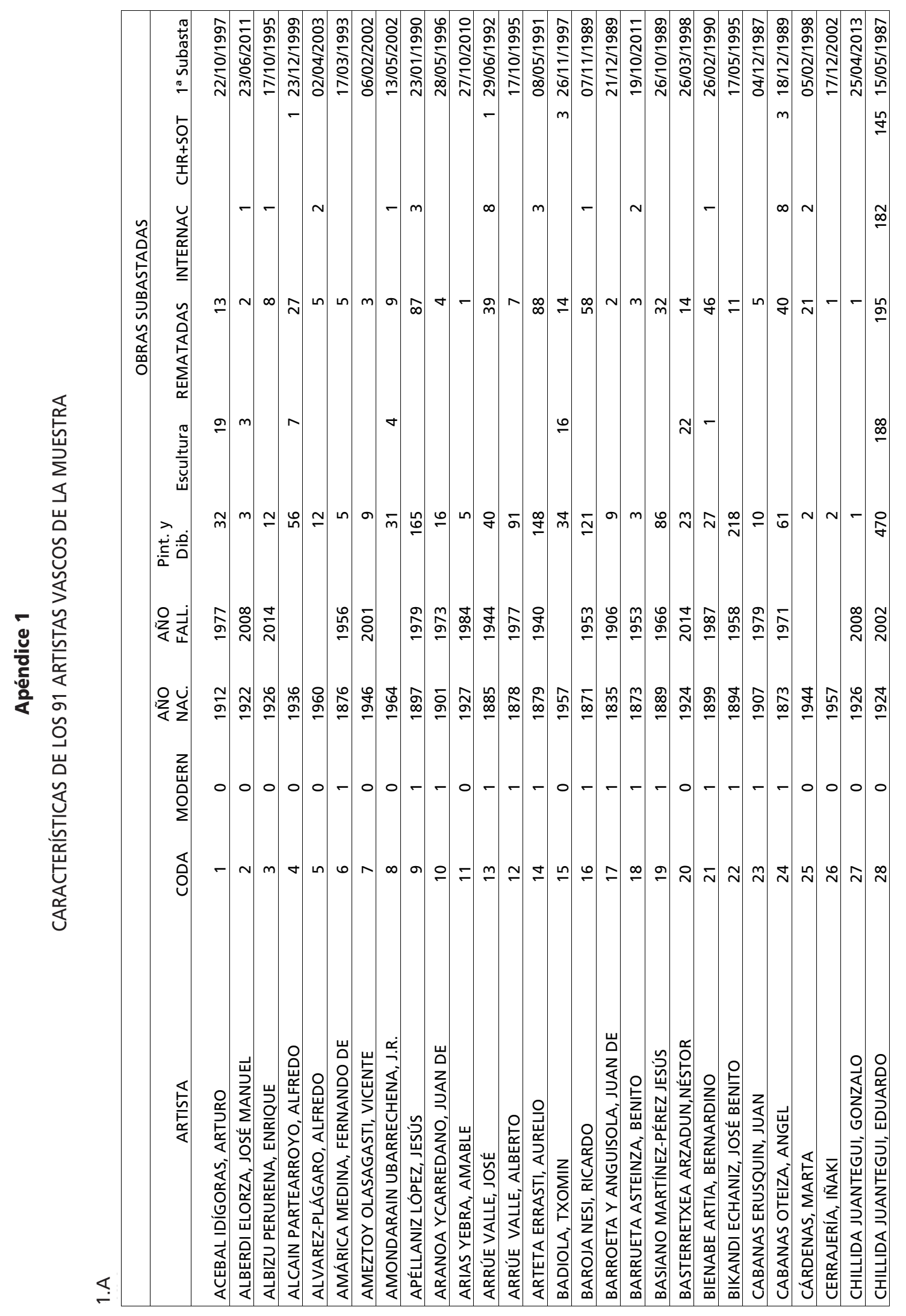




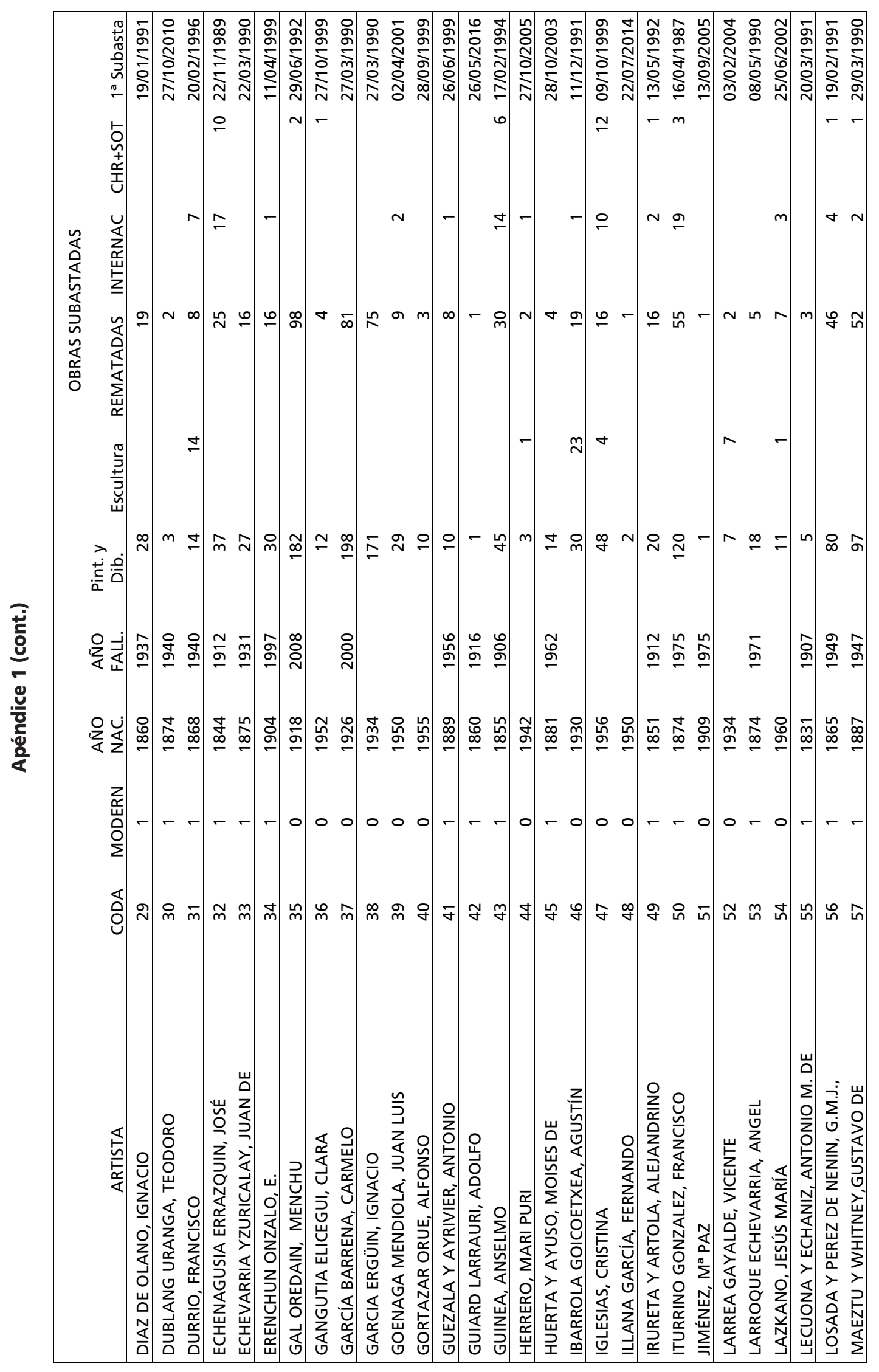




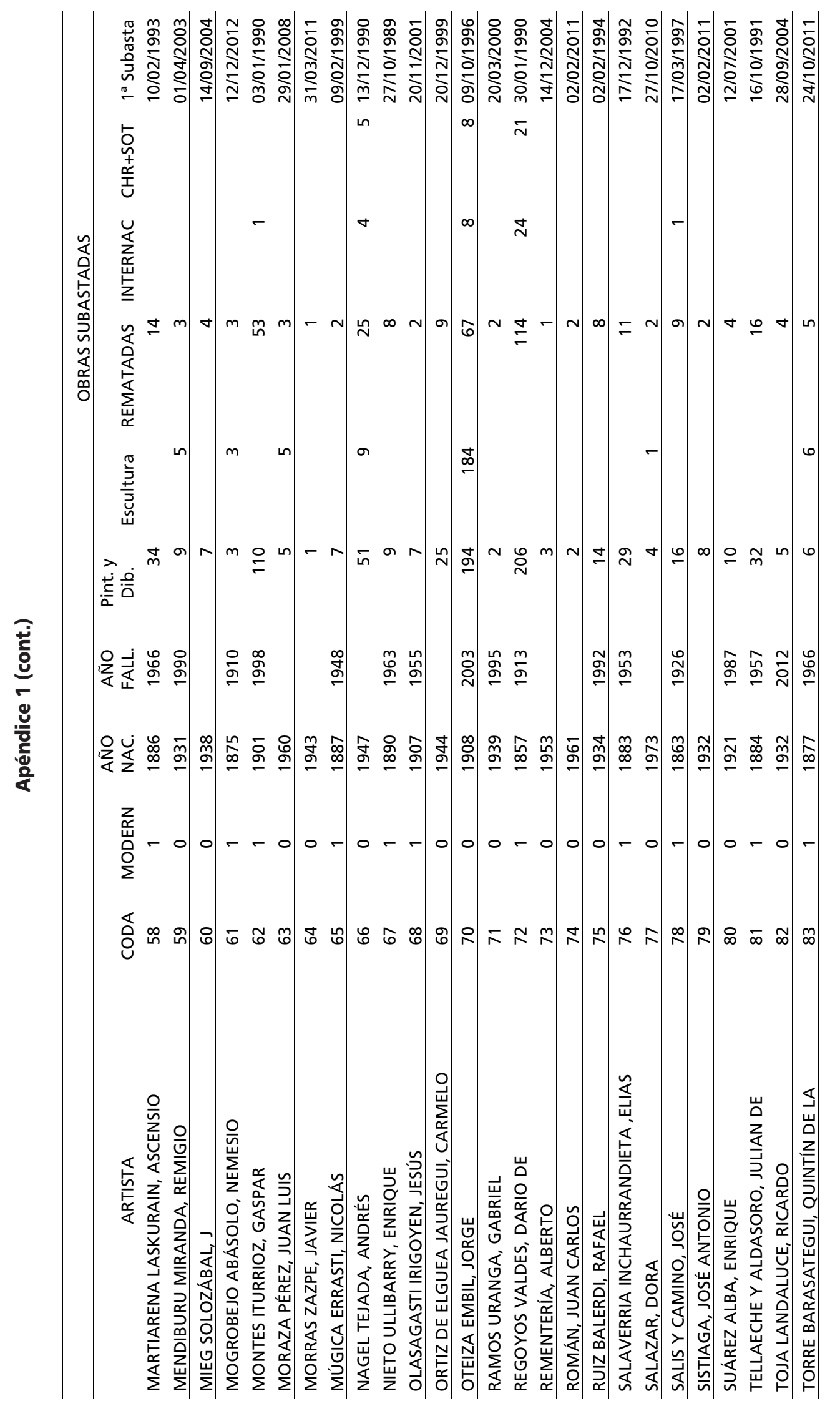




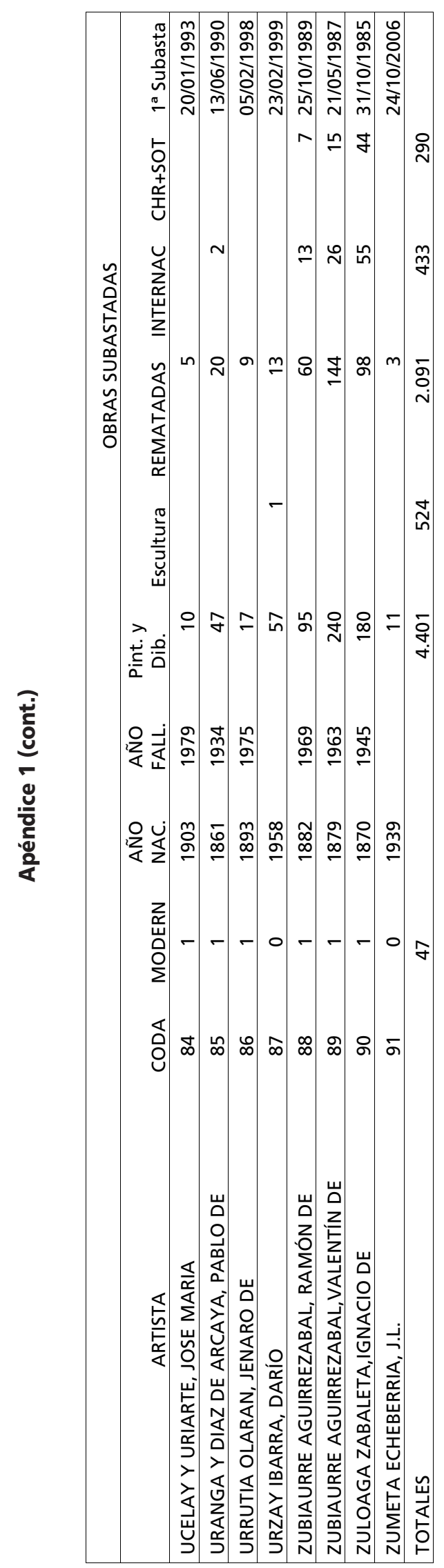




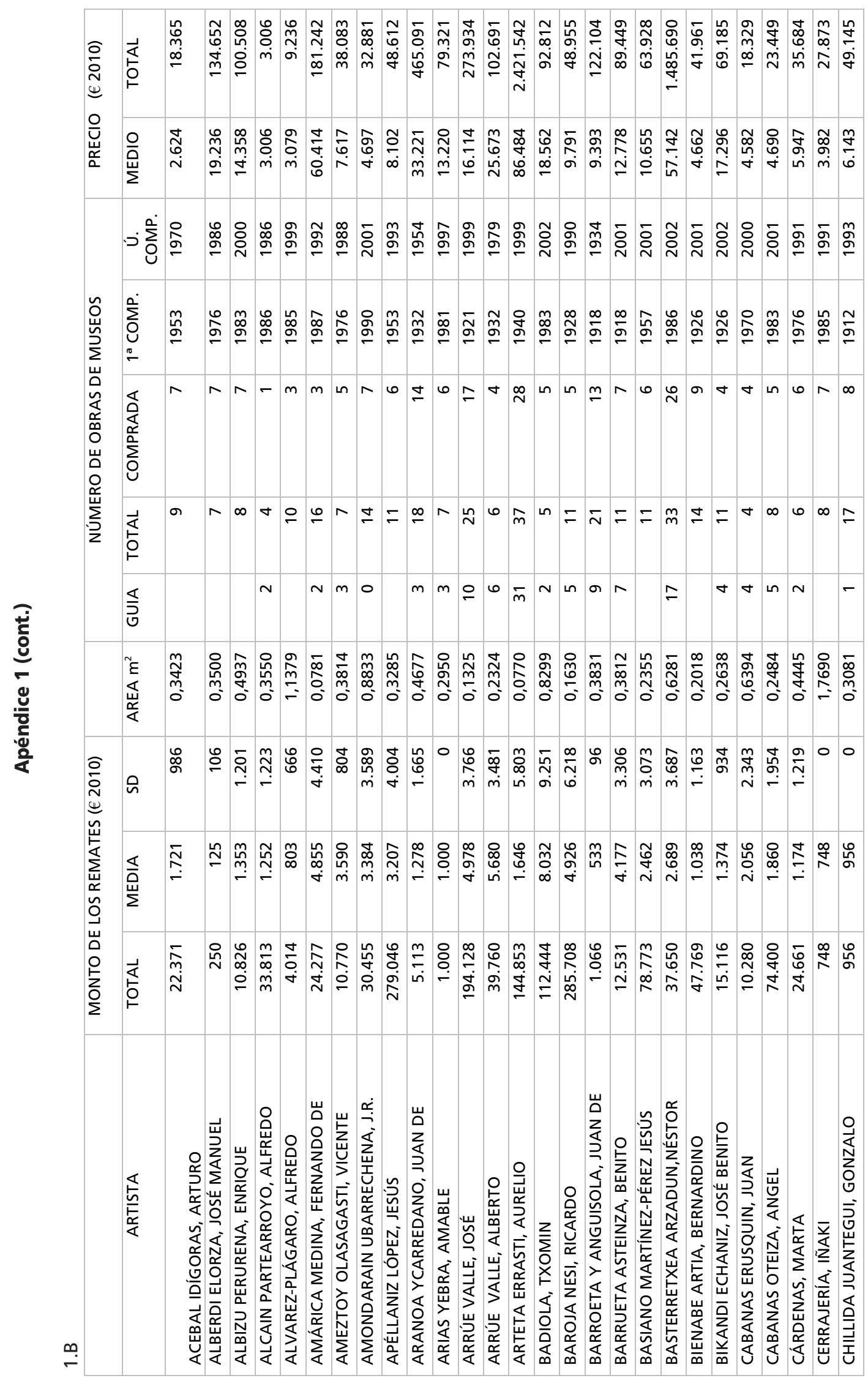




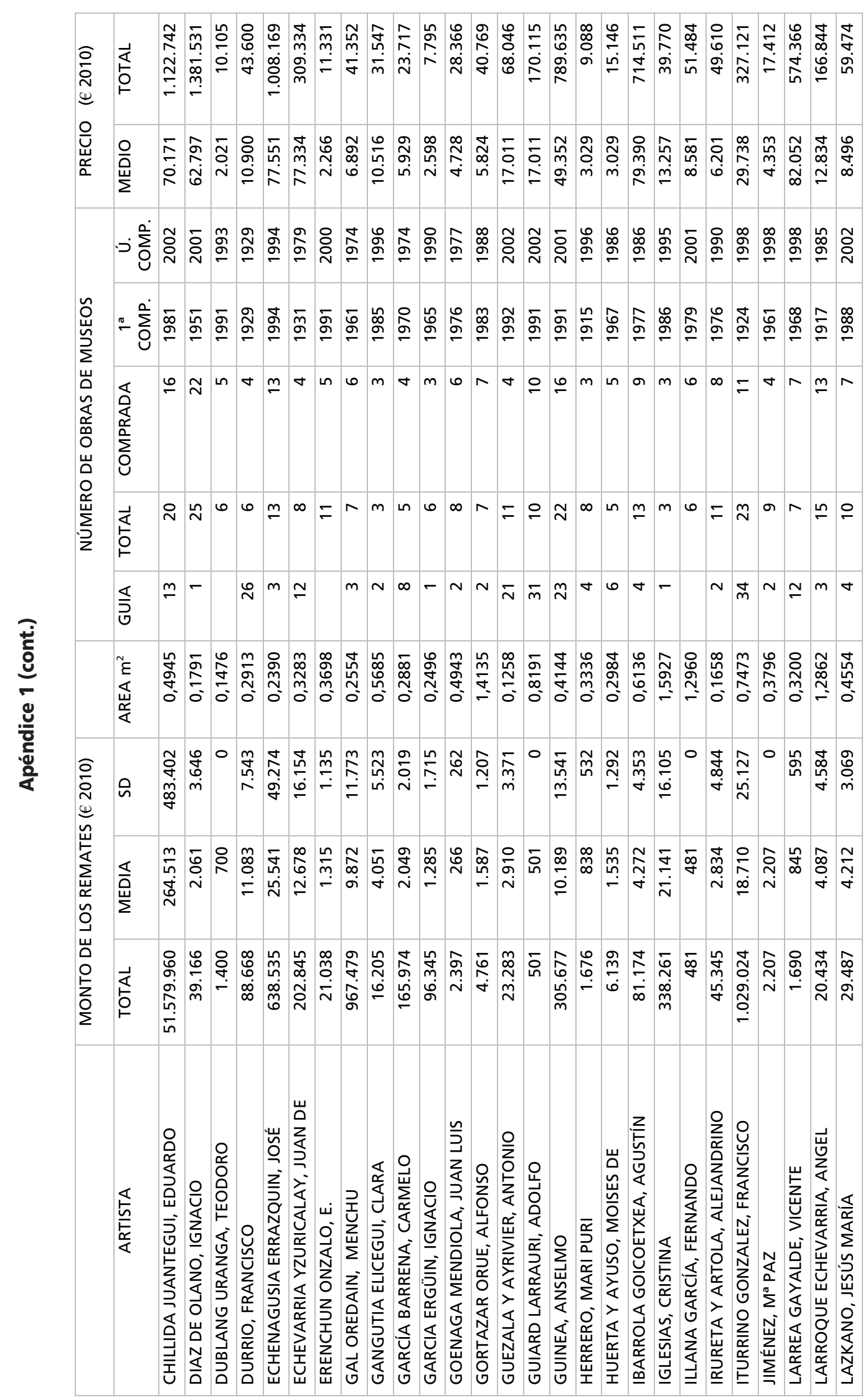




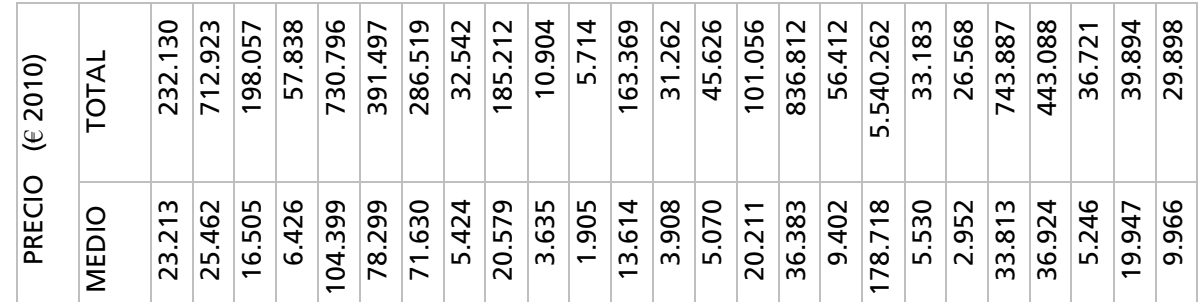

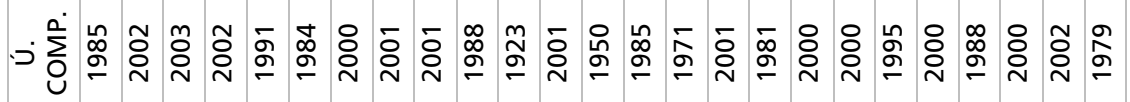

芫

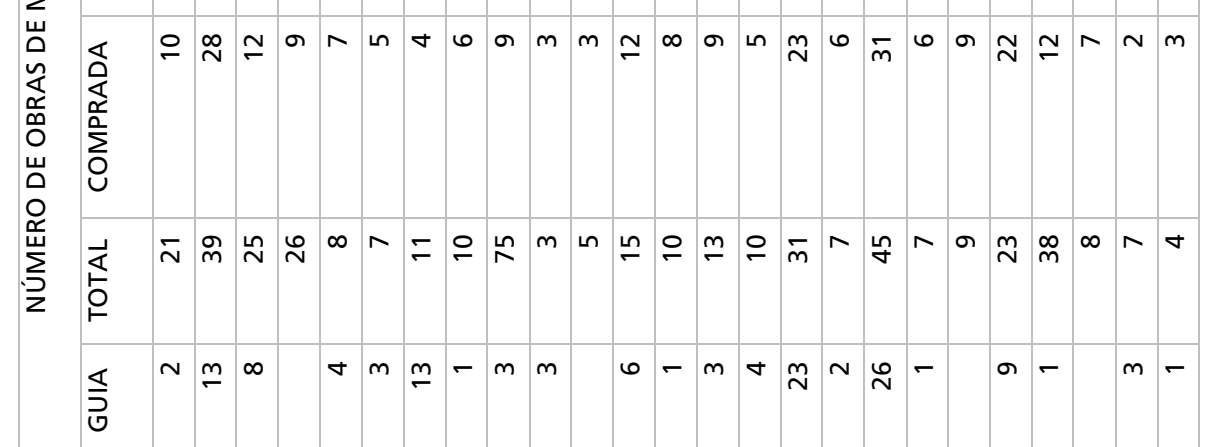

₹

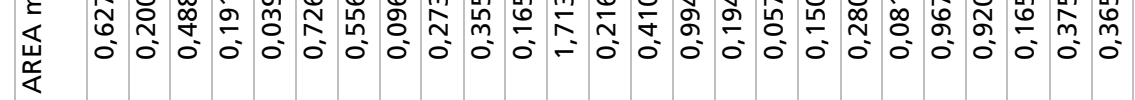

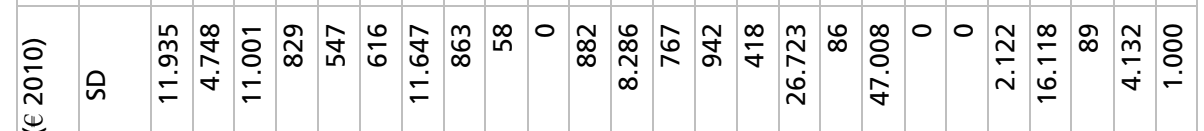
崖

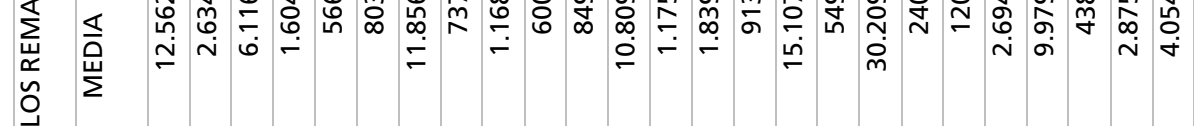

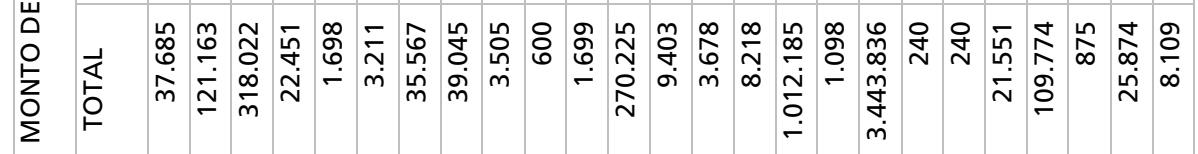

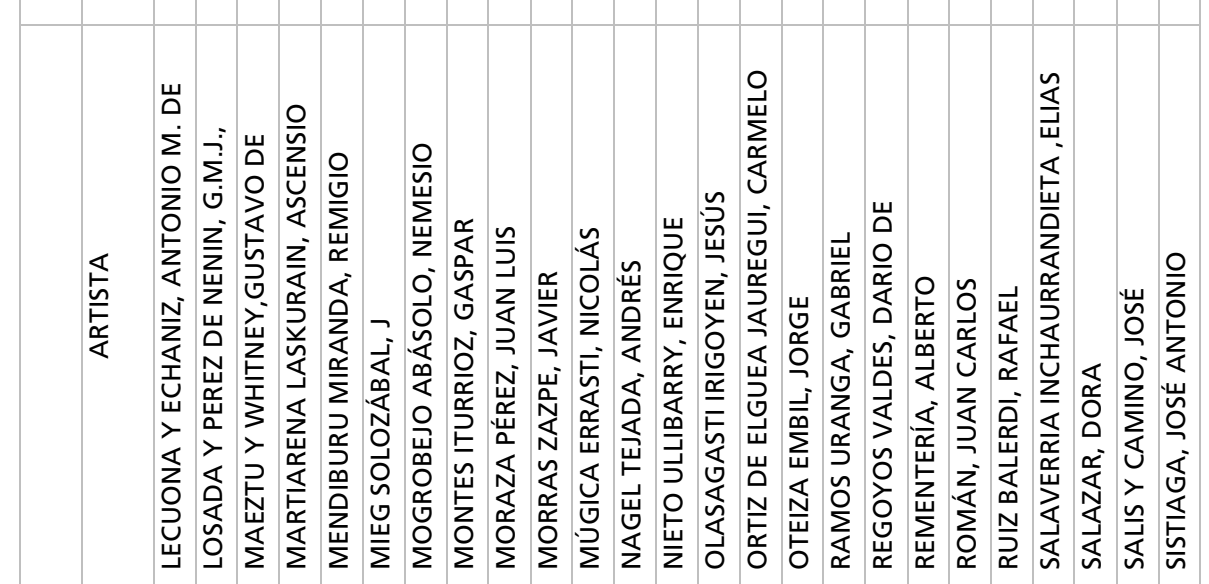




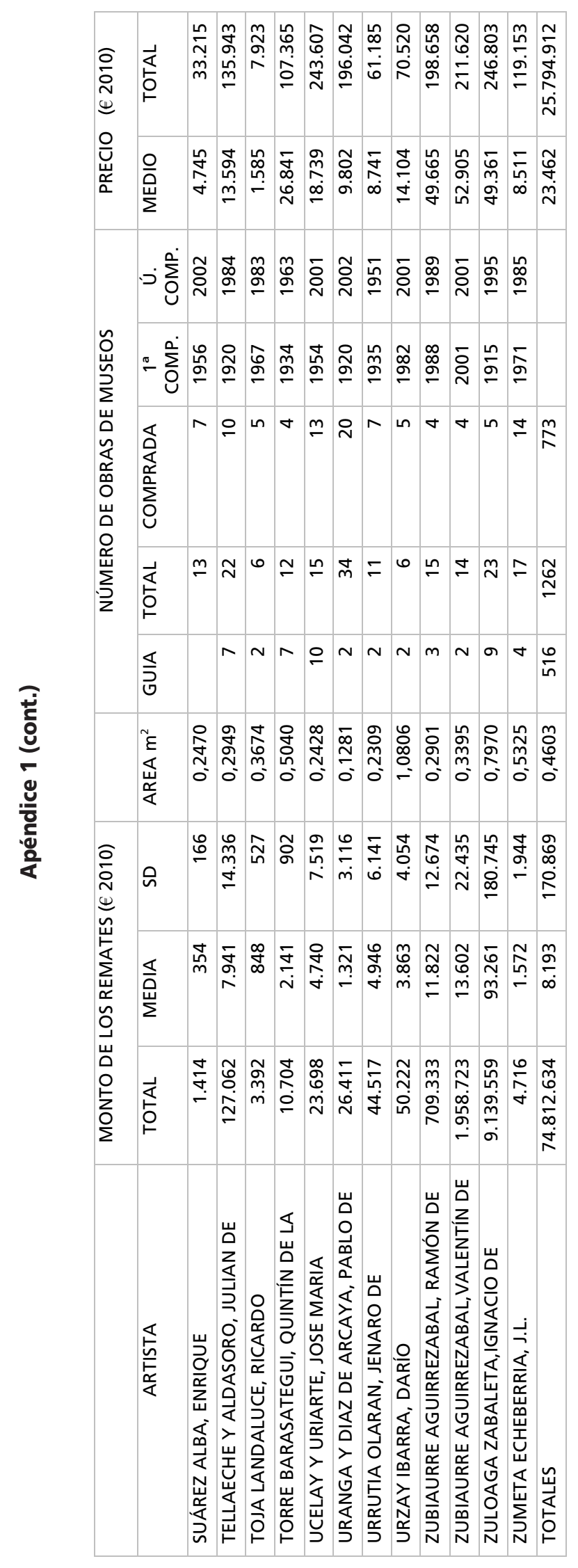

